\title{
Chemical, Nutritional and Organoleptical Characteristics of Orange-Based Formulated Low-Calorie Jams
}

\author{
Rafeek M. Abolila, Hassan Barakat, Hassan A. El-Tanahy, Hamdy A. El-Mansy \\ Food Technology Department, Faculty of Agriculture, Benha University, Banha, Egypt \\ Email: "hassan.barakat@fagr.bu.edu.eg
}

Received 29 July 2015; accepted 25 October 2015; published 28 October 2015

Copyright (C) 2015 by authors and Scientific Research Publishing Inc.

This work is licensed under the Creative Commons Attribution International License (CC BY). http://creativecommons.org/licenses/by/4.0/

(c) $\underset{\mathrm{EY}}{\mathrm{P}}$ Open Access

\begin{abstract}
Twelve low-calorie orange-based formulated jams were prepared mainly from orange, pumpkin and papaya, and then sweetened using fructose (F), stevioside (St) and sucralose (Su). The nutritional value of formulated jams was estimated after the approximate chemical composition and total soluble solid have been determined. The effect of storage on total phenolic compounds, antioxidant capacity, carotenoids content and vitamin $C$ was investigated. Organoleptical attributes of prepared low-calorie jams were done as well. Results indicated that the nutritive value [kcal 100

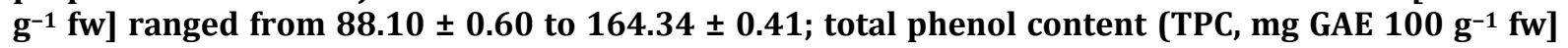
ranged from $188.52 \pm 2.45$ to $411.79 \pm 3.3$; the antioxidant capacity $\left(\mu \mathrm{mol}\right.$ TE $\left.\mathrm{g}^{-1} \mathrm{fw}\right)$ ranged from $14.57 \pm 0.86$ to $32.39 \pm 1.19$; total carotenoids $\left[\mathrm{mg}^{100} \mathrm{~g}^{-1} \mathrm{fw}\right.$ ] ranged from $115.20 \pm 5.66$ to 204.33 \pm 4.21 ; vitamin $\mathrm{C}$ [mg $100 \mathrm{~g} \mathrm{fw}$ ] ranged from $8.94 \pm 1.07$ to $28.77 \pm 4.46$; total soluble solids [Brix] ranged from $22.53 \pm 0.05$ to $43.37 \pm 0.13$. Jams storage for a period of 12 months at room temperature led to a decrease in vitamin C content, TPC, antioxidant capacity, while total soluble solids (TSS) and carotenoids increased during storage. The results of organoleptical attributes showed that the formulas 011,01 had the highest score of color and odor respectively while 07 recorded maximum score for taste, texture and bitterness respectively. Statistical analysis showed that storage intervals and treatments had a significant $(p<0.05)$ effect on sensory quality of diet jam. The organoleptical characteristics were affected with the extension of shelf-life. Addition of stevioside and sucralose to formulate the low-calorie jam increased total phenol and antioxidant capacity, improved color, taste and produced targeted low-calorie jams. The use of sweeteners such as fructose, sucralose and stevioside in the manufacture of orange diet jam was shown to be satisfactory, resulting in low-calorie jams, improved the quality and could be produced commercially.
\end{abstract}

\section{Keywords}

Low-Calorie Jam, Chemical, Nutritional, Organoleptical Characteristics

\footnotetext{
${ }^{*}$ Corresponding author.
}

How to cite this paper: Abolila, R.M., Barakat, H., El-Tanahy, H.A. and El-Mansy, H.A. (2015) Chemical, Nutritional and Organoleptical Characteristics of Orange-Based Formulated Low-Calorie Jams. Food and Nutrition Sciences, 6, 1229-1244. http://dx.doi.org/10.4236/fns.2015.613129 


\section{Introduction}

Recently, health concerns associated with high sugar intake include excessive calorie consumption and related diseases are considered as crucial issues for many foods organizations [1]. Several organizations recommend consumption of fruits and vegetables as well as reduce total calories intake [2]-[4]. The growing concern with health and higher incidence of obesity, metabolic syndrome and diabetes has resulted in an increase in interest of low-calorie food consumption [5] [6]. In this context, consumption of low calories and light products which are indicated for peoples with diabetic or other medical restrictions, including obesity was increased [7] as well as for aesthetics and health concerned peoples. With increasing of consumer interest in reducing sugar intake, food products made with sweeteners rather than sugar have become more popular and depleted quickly with high market share [8].

Surely, the production of low-calorie products must comprise low-calorie raw materials and low-calorie sweeteners [9]. To meet the recommended reduction of calories, several foods have been introduced into the market as low-calorie products incorporating natural and/or artificial sweeteners. Low-calorie sweeteners (LCSs) are added to many foods and beverages, for reducing total calories, while maintaining palatability [10]. LCSs have only begun to develop over the past 30 years, concomitant with the increase in obesity and type 2 diabetes, which led to an increased interest in methods of losing weight or maintaining weight loss [11] [12]. The LCSs currently licensed for use in many countries [13]. The varying chemical properties of each LCS mean that they are suited to diverse uses and wide applications could be presented [12]. Typically, the energy difference between regular and LCS-sweetened products is more pronounced in beverages, processed fruits and vegetables more than foods [14] [15].

Interestingly, sucralose is the only commercial sweetener derived from sucrose and is an intense sweetener made by selective substitution of the hydroxyl groups of sucrose with chlorine [16]. It can be used in cooking and baking and in soft drinks, tea, coffee and chilled desserts. Sucralose is non-caloric, noncariogenic and has no effect on blood glucose or insulin levels. It has a taste profile very close to sucrose, presenting very low level of bitterness and sourness. The use of sucralose, one of the newest sweeteners of high sweetening power, has been gradually increasing [17]-[21].

Stevia is a natural sweetener, extracted from leaves of the plant (Stevia rebaudiana Bert.), and produces diterpene glycosides that are low-calorie sweeteners. Stevia extracts, besides having therapeutic properties, contain a high level of sweetening compounds, known as steviol glycosides [22] [23]. Stevia contains intensely sweet substances that are 250 to 300 times sweeter than sugar [24]. Steviol glycosides are safe (GRAS) by the FDA. Steviol glycosides can be particularly beneficial to those suffering from obesity, diabetes mellitus, heart disease and dental caries [25]. Production of low-calorie jams could be an important issue for many people groups who suffer from obesity, diabetics, sugar allergic, and dental decay. In addition, it will be a satisfaction to consumers, who maintain their health or for weight management programs.

Nowadays the dietary awareness of consumers has led to the growth of health food industry, and thus alternative jams containing artificial sweeteners should be available. According to the Egyptian specification 2005, there are two kinds of low-calorie jams: 1) contain not less than 200 calorie per $100 \mathrm{gm}$ and 2) should not be less than 100 calorie per 100 gm with shelf-life stability for one year. Recently, Muhammad et al. [26], Kopjar et al. [27], Tamer et al. [28], Basu et al. [29], Gao et al. [30], Kerdsup et al. [31], Youssef et al. [32], Teangpook et al. [33], Levaj et al. [34] investigated many jams formulas sweetened with different LCSs and approved that there are no drastic effects on prepared jam characteristics.

Therefore, the current work is aimed at determining the chemical and nutritional properties of the prepared low-calorie jams incorporated with low-calorie sweeteners compared with the common sweetened jams. The microbiological characteristics of prepared low-calorie jams and sensory attractiveness of prepared low-calorie jams are assessed. Moreover, the study of the effect of storage period on chemical and nutritional properties, microbiological and sensory characteristics of low-calorie jams was investigated.

\section{Materials and Methods}

\subsection{Fresh Fruits}

The raw materials used for preparing low calories jams are: orange (Citrus sinensis), fully mature Egyptian baladi orange fruits and pumpkin (Cucurbita maxima) obtained from local market at Qaliuobia Governorate, Egypt. Papaya fruits (Carica papaya L. cv. Sunrise Solo), $750 \mathrm{~g}$ to $1750 \mathrm{~g}$ /each were obtained from farm of Faculty of 
Agriculture, Moshtohor Benha Univ., Egypt. The fruit surface was treated by $\mathrm{H}_{2} \mathrm{O}_{2} 5 \%$ as disinfectant then ripened at room temperature for 3 - 4 days.

\subsection{Fixed Ingredients}

Fructose was obtained from the National Company for Maize Products, $10^{\text {th }}$ Ramadan city, A1, Egypt. LM pectin (E 440 (a) — LM 104 AS-FS Pectin, Food grade, Denemark), potassium sorbate (E 202—Food grade, China), citric acid (E 330-Food grade, China), calcium lactate (E 327-Food grade, Belgum), sodium benzoate (E 211-Food grade, China) were obtained from Hero factory for jams and beverages manufacturing, Tersa, Qaluiobia, Egypt. Stevioside and sucralose (Fineprint company) imported by Rebat company for food stuffs trade, Egypt.

\subsection{Fruits Preparation}

Orange fruits were washed, capsules were removed and external layer had been removed using the carborandum then cut into halves and extracted. Afterword, extracted juice sterilized at $90^{\circ} \mathrm{C}$ for 5 min then cooled down to $45^{\circ} \mathrm{C}$. Exactly $1 \mathrm{~kg}$ was filled in a polyethylene bag and sealed after removing the air then kept under $-20^{\circ} \mathrm{C}$. Peel's halves were cut by sharp knife into small slices then boiled into hot water $100^{\circ} \mathrm{C}$ for $1 \mathrm{~h}$, drained, cooled down, filled in a polyethylene bag then kept under $-20^{\circ} \mathrm{C}$. Papaya fruit were washed, peeled, cut into small cubic and homogenized by kitchen machine grounder (SIEMENS, type CNCM11ST Germany). Exactly, $1 \mathrm{~kg}$ was filled in a polyethylene bag, sealed then kept under $-20^{\circ} \mathrm{C}$. Pumpkin fruits were washed, peeled, cut into small cubes, steam blanched for 10 min, cooled down, homogenized by kitchen machine grounder, filled in a polyethylene bag, sealed then kept under $-20^{\circ} \mathrm{C}$.

\subsubsection{Formulation of Low-Calorie Jams}

Many preliminary experiments have been done for selecting the best fruits and sweeteners portions. Twelve jam formulas were prepared according to Table 1.

Table 1. Low calories orange-based formulated jams.

\begin{tabular}{|c|c|c|c|c|c|c|c|c|c|c|c|}
\hline \multirow{3}{*}{ 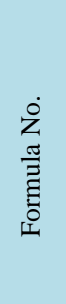 } & \multicolumn{11}{|c|}{ Ingredients } \\
\hline & \multicolumn{4}{|c|}{ Fruits puree (\%) } & \multicolumn{3}{|c|}{ Sweeteners (\%) } & \multicolumn{4}{|c|}{ Additives (\%) } \\
\hline & $\begin{array}{l}\text { वे } \\
\text { జ్రే }\end{array}$ & $\frac{\Xi}{\bar{\Xi}}$ & 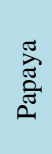 & 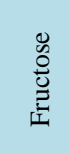 & 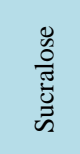 & 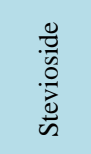 & 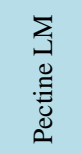 & 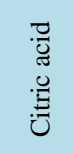 & $\begin{array}{l}\frac{\pi}{0} \\
\frac{\pi}{\pi} \\
\tilde{U}\end{array}$ & 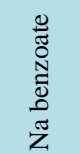 & 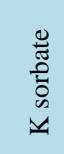 \\
\hline $\mathrm{O} 1$ & 100 & - & - & 100 & - & - & 0.75 & 0.35 & 0.15 & 0.01 & 0.06 \\
\hline $\mathrm{O} 2$ & 100 & - & - & 75 & 16.5 & 8.50 & 0.9 & 0.35 & 0.15 & 0.01 & 0.06 \\
\hline O3 & 100 & - & - & 50 & 33.5 & 16.50 & 0.9 & 0.35 & 0.15 & 0.01 & 0.06 \\
\hline $\mathrm{O} 4$ & 100 & - & - & 25 & 50 & 25 & 1 & 0.35 & 0.15 & 0.01 & 0.06 \\
\hline O5 & 75 & 25 & - & 100 & - & - & 0.75 & 0.35 & 0.15 & 0.01 & 0.06 \\
\hline O6 & 75 & 25 & - & 75 & 16.5 & 8.50 & 0.9 & 0.35 & 0.15 & 0.01 & 0.06 \\
\hline $\mathrm{O} 7$ & 75 & 25 & - & 50 & 33.5 & 16.50 & 0.9 & 0.35 & 0.15 & 0.01 & 0.06 \\
\hline O8 & 75 & 25 & - & 25 & 50 & 25 & 1 & 0.35 & 0.15 & 0.01 & 0.06 \\
\hline O9 & 75 & - & 25 & 100 & - & - & 0.75 & 0.37 & 0.15 & 0.01 & 0.06 \\
\hline $\mathrm{O} 10$ & 75 & - & 25 & 75 & 16.5 & 8.50 & 0.9 & 0.37 & 0.15 & 0.01 & 0.06 \\
\hline O11 & 75 & - & 25 & 50 & 33.5 & 16.50 & 0.9 & 0.37 & 0.15 & 0.01 & 0.06 \\
\hline $\mathrm{O} 12$ & 75 & - & 25 & 25 & 50 & 25 & 1 & 0.37 & 0.15 & 0.01 & 0.06 \\
\hline
\end{tabular}




\subsubsection{Procedure}

Combinations of fruit puree were heated until boiling, LMP (104 AS-FS) food grade Pectin (E 440a), Denmark was added as $0.75 \%-1 \%$ [35] to fruits mix and allowed to boil for $10 \mathrm{~min}$ until all pectin dissolved. Sucralose, fructose, and stevioside were added followed by calcium lactate $(0.15 \%)$. The mixture was boiled until desired concentration. Finally, citric acid, sodium benzoate and potassium sorbate $(0.06 \%)$ were added, stirred for 1 min, and hot-filed in sterile jars. After $24 \mathrm{~h}$, formulated jams were subjected to whole analysis.

\subsubsection{Analytical Methods}

Nutritional value: The nutritional value of different fresh and fried vegetarian diets was calculated basically on the crude protein, lipids and carbohydrates data according to [36].

Ascorbic acid determination: Ascorbic acid determination using the 2,6-dichlorophenol indophenol dye by titration method according to [36].

Determination of carotenoids: Carotenoids were determined in the acetonic extract and expressed as mg 100 $\mathrm{g}^{-1} \mathrm{fw}$ according to [37]

Determination of total phenolic compounds: After extraction of total phenolic compound, concentration of TPC was determined by Folin-Ciocalteau method. After $1 \mathrm{~h}$ at ambient temperature, the absorbance was measured at $765 \mathrm{~nm}$ and The TPC was expressed as milligram gallic acid equivalents per gram sample (mg GAE 100 $\mathrm{g}^{-1} \mathrm{fw}$ ) according to [38].

\subsubsection{Determination of Radical DPPH-Scavenging Activity}

Antioxidant activity was measured spectrophotometrically using the 2,2-diphenylpicrylhy-drazyl (DPPH) radical. According to this method, extracted samples, which were made to react with the radical solution and rest for 30 minutes at room temperature, were measured for absorbance at $517 \mathrm{~nm}$, and the inhibition percentage of DPPH free radical was calculated [39].

\subsubsection{Organoleptical Attributes}

Organoleptical attributes of the different formulas was carried out. Forty panelists of the staff members and students from the Food Science Department, Faculty of Agriculture, Benha University, in the age range of 20 to 57 years were asked to evaluate the prepared jams towards color, taste, odor, texture, and bitterness. A 7-point hedonic scale ( 7 being like extremely, 4 like accepted and 1 dislike extremely) was used. Results were subjected to analysis of variance and average of the mean values of the aforementioned attributes and their standard error were calculated according to Wilson et al. [40].

\subsubsection{Statistical Analysis}

The statistical analysis was carried out using SPSS program (ver. 19) with multi-function utility regarding to the experimental design under significance level of 0.05 for the whole results and multiple comparisons were carried out applying LSD with Duncan according to Steel et al. [41].

\section{Results and Discussion}

\subsection{Nutritive Value of Prepared Jams}

Twelve low calorie orange-based formulated jams were prepared mainly from orange, pumpkin and papaya then sweetened using fructose (F), stevioside (St) and sucralose (Su) as mentioned in Table 1. In order to calculate the actual nutritional value of prepared jams, the proximate chemical composition was determined (data not presented). Comparing between mean of storage period for all formulas the nutritive value ranged from $88.10 \pm$

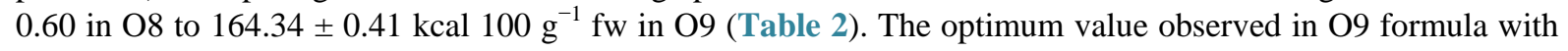
(100:0, F:St + Su) while the minimum value observed in O8 formula with (25:75, F:St + Su). Data indicated that the formulas O4, O8 and O12 with (25:75) ratio meet the required specifications of 2005's Egyptian standards

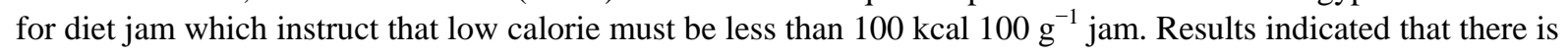
a significant difference among all formulas regarding the different ratios of fructose replacement by sweeteners. These results are in agreement with Salvador et al. [42] who demonstrated that caloric value of the low calorie yacon jams was ranged from 116.4 to $140.0 \mathrm{kcal} 100 \mathrm{~g}^{-1}$. The orange and orange mixed jams reduced caloric value and can be considered as light or "low calorie" products. The high sweetening ability of the sugars present 
Table 2. Nutritional value (kcal $100 \mathrm{~g}^{-1} \mathrm{fw}$ ) of orange-based formulated low calories jams sweetened with fructose, stevioside and sucralose during 12 months of storage (mean $\pm \mathrm{SD}$ ).

\begin{tabular}{|c|c|c|c|c|c|c|c|c|}
\hline \multirow{2}{*}{ Fruit type } & \multirow{2}{*}{$\begin{array}{l}\text { Sweetener } \\
\text { formula }\end{array}$} & \multicolumn{5}{|c|}{ Storage period (month) } & \multirow{2}{*}{$\begin{array}{c}\text { Mean of storage } \\
\text { period }\end{array}$} & \multirow{2}{*}{$\begin{array}{l}\text { Mean of } \\
\text { fruit type }\end{array}$} \\
\hline & & 0 & 3 & 6 & 9 & 12 & & \\
\hline \multirow{4}{*}{ Orange } & $\mathrm{O} 1$ & $167 \pm 0.04$ & $163.95 \pm 0.15$ & $165.07 \pm 0.24$ & $164.03 \pm 0.07$ & $161.21 \pm 0.14$ & $164.25 \pm 0.20 \mathrm{i}$ & \multirow{4}{*}{$\begin{array}{c}131.94 \pm \\
2.34 \mathrm{~b}\end{array}$} \\
\hline & $\mathrm{O} 2$ & $145.82 \pm 0.21$ & $152.32 \pm 0.13$ & $147.5 \pm 0.28$ & $141.63 \pm 0.23$ & $143.81 \pm 0.21$ & $146.21 \pm 0.36 \mathrm{~g}$ & \\
\hline & $\mathrm{O} 3$ & $119.72 \pm 0.29$ & $130.9 \pm 0.54$ & $121.79 \pm 0.07$ & $125.61 \pm 0.27$ & $118.96 \pm 0.15$ & $123.40 \pm 0.49 e$ & \\
\hline & $\mathrm{O} 4$ & $88.18 \pm 0$ & $95.59 \pm 0.57$ & $96.6 \pm 0.1$ & $96.26 \pm 0.15$ & $92.94 \pm 0.27$ & $93.92 \pm 0.42 b$ & \\
\hline \multirow{4}{*}{$\begin{array}{l}75 \% \text { orange/ } \\
25 \% \text { pumpkin }\end{array}$} & O5 & $150.88 \pm 0.27$ & $156.35 \pm 0.39$ & $153.29 \pm 0.07$ & $155.69 \pm 0.14$ & $153.43 \pm 0.05$ & $153.93 \pm 0.25 h$ & \multirow{5}{*}{$\begin{array}{c}124.35 \pm \\
2.31 \mathrm{a}\end{array}$} \\
\hline & O6 & $139.18 \pm 0.3$ & $146.41 \pm 0.4$ & $134.93 \pm 0.25$ & $133.36 \pm 0.2$ & $141.38 \pm 0.3$ & $139.05 \pm 0.48 f$ & \\
\hline & $\mathrm{O} 7$ & $102.94 \pm 0.23$ & $125.32 \pm 0.4$ & $115.38 \pm 0.2$ & $118.02 \pm 0.2$ & $119.85 \pm 0.47$ & $116.30 \pm 0.76 d$ & \\
\hline & O8 & $81.55 \pm 0.29$ & $94.92 \pm 0.1$ & $87.44 \pm 0.28$ & $93.09 \pm 0.25$ & $83.5 \pm 0.05$ & $88.10 \pm 0.60 \mathrm{a}$ & \\
\hline \multirow{4}{*}{$\begin{array}{l}75 \% \text { orange/ } \\
25 \% \text { papaya }\end{array}$} & O9 & $166.66 \pm 0.14$ & $170.57 \pm 0.13$ & $160.73 \pm 0.37$ & $157.36 \pm 0.02$ & $166.4 \pm 0.01$ & $164.34 \pm 0.41 \mathrm{i}$ & \\
\hline & $\mathrm{O} 10$ & $132.94 \pm 0.23$ & $154.08 \pm 0.36$ & $146.76 \pm 0.06$ & $149.86 \pm 0.09$ & $144.67 \pm 0.19$ & $145.66 \pm 0.64 \mathrm{~g}$ & \multirow{3}{*}{$\begin{array}{c}132.6 \pm \\
2.26 b\end{array}$} \\
\hline & $\mathrm{O} 11$ & $113 \pm 0.3$ & $130.53 \pm 0.51$ & $119.29 \pm 0.15$ & $126.09 \pm 0.16$ & $125.4 \pm 0.17$ & $122.86 \pm 0.62 \mathrm{e}$ & \\
\hline & $\mathrm{O} 12$ & $92.08 \pm 0.09$ & $106.91 \pm 0.16$ & $94.99 \pm 0.22$ & $94.8 \pm 0.09$ & $98.9 \pm 0.68$ & $97.54 \pm 0.61 c$ & \\
\hline \multicolumn{2}{|c|}{ Mean of total storage period } & $125.00 \pm 2.61 \mathrm{~A}$ & $135.65 \pm 2.19 \mathrm{C}$ & $128.65 \pm 2.29 \mathrm{~B}$ & $129.65 \pm 2.16 \mathrm{~B}$ & $129.21 \pm 2.33 \mathrm{~B}$ & & \\
\hline \multicolumn{2}{|c|}{$\begin{array}{c}\text { Ratio of } \\
\text { (fructose:sweeteners) }\end{array}$} & $\begin{array}{l}\mathrm{O}(1,5,9) \\
(100 / 0)\end{array}$ & $\begin{array}{l}\mathrm{O}(2,6,10) \\
(75 / 25)\end{array}$ & $\begin{array}{c}\mathrm{O}(3,7,11) \\
(50 / 50)\end{array}$ & $\begin{array}{l}\mathrm{O}(4,8,12) \\
(25 / 75)\end{array}$ & & & \\
\hline \multicolumn{2}{|c|}{ Mean of sweetener formula } & $160.84 \pm 0.49 \mathrm{D}$ & $143.64 \pm 0.57 \mathrm{C}$ & $120.85 \pm 0.69 B$ & $93.18 \pm 0.68 \mathrm{~A}$ & & & \\
\hline \multirow{2}{*}{ LSD at 0.05} & Storage (S) & Fruit (F) & Sweetener (T) & $\mathrm{S}^{*} \mathrm{~F}$ & $\mathrm{~S}^{*} \mathrm{~T}$ & $\mathrm{~F}^{*} \mathrm{~T}$ & $\mathrm{~S} * \mathrm{~F}^{*} \mathrm{~T}$ & \\
\hline & 1.34 & 1.20 & 1.20 & 2.33 & 2.69 & 2.08 & 4.65 & \\
\hline
\end{tabular}

a, b \& c: There is no significant difference $(\mathrm{P}>0.05)$ between any two means, within the same column have the same superscript letter. $n=3$. A, B \& $\mathrm{C}$ : There is no significant difference $(\mathrm{P}>0.05)$ between any two means for the same attribute within the same row have the same superscript letter.

in orange, mainly fructose [43], contributed to the preparation of a jam with low addition of sucrose and therefore low content of calories occurred. Comparing between mean of total storage period, results indicated that increasing in nutritive value during storage period. The cause of increasing nutritive value may be due to increasing the soluble carbohydrates during storage. Mean of sweetener formula indicated that maximum nutritive val-

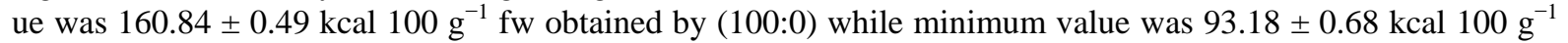
fw obtained by (25:75) and there is a significant difference between all ratios. Yuyama et al. [44] found reduction in the caloric value ranged from $25.9 \%$ to $37.9 \%$ in jambolão fruit jams using four types of sweeteners individually or in combination (saccharin, cyclamate, acesulfame and St). Moreover, [45] [46] reported that stevioside give low calories.

\subsection{Total Phenolic Compounds and Antioxidant Capacity}

Phenolics are naturally occurring compounds widely distributed in the plant kingdom and beneficial components of human daily diet Le et al. [47]. Presented data in Table 3, indicated that orange jams had the highest score for total phenol compounds (TPC) which was311.24 $\pm 4.95 \mathrm{mg}$ GAE $100 \mathrm{~g}^{-1}$ followed by $284.18 \pm 5.82 \mathrm{mg}$ GAE $100 \mathrm{~g}^{-1}$ in OY jams while minimum score was $252.19 \pm 4.63 \mathrm{mg}$ GAE $100 \mathrm{~g}^{-1}$ in OK jams. Tamer et al. [28]

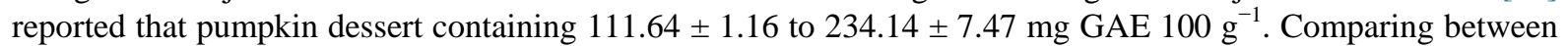
mean of storage period after 12 month the results indicated that maximum TPC was $411.79 \pm 3.31$ mg GAE 100 $\mathrm{g}^{-1}$ in $\mathrm{O} 4$ while minimum total phenol was $188.52 \pm 2.45 \mathrm{mg}$ GAE $100 \mathrm{~g}^{-1}$ in O9. The results illustrated that the TPC increased with decreasing the fructose. These results are in agreement with Istratii et al. [48] who found TPC in goji fruits jam and jelly was $351 \pm 7.25 \mathrm{mg}$ GAE $100 \mathrm{~g}^{-1}$. Comparing between mean of total storage period, results indicated that TPC decreased from $274.4 \pm 4.59 \mathrm{mg} \mathrm{GAE} 100 \mathrm{~g}^{-1}$ at zero time to $251.04 \pm 4.94 \mathrm{mg}$ GAE $100 \mathrm{~g}^{-1}$ after 9 months during storage at room temperature. These results are in agreement with [49]-[52] 
Table 3. Total phenol content (mg GAE $100 \mathrm{~g}^{-1} \mathrm{fw}$ ) of orange-based formulated low calories jams sweetened with fructose, stevioside and sucralose during 12 months of storage (mean $\pm \mathrm{SD}$ ).

\begin{tabular}{|c|c|c|c|c|c|c|c|c|}
\hline \multirow{2}{*}{ Fruit type } & \multirow{2}{*}{$\begin{array}{l}\text { Sweetener } \\
\text { formula }\end{array}$} & \multicolumn{5}{|c|}{ Storage period (month) } & \multirow{2}{*}{$\begin{array}{c}\text { Mean of storage } \\
\text { period }\end{array}$} & \multirow{2}{*}{$\begin{array}{l}\text { Mean of } \\
\text { fruit type }\end{array}$} \\
\hline & & 0 & 3 & 6 & 9 & 12 & & \\
\hline \multirow{5}{*}{ Orange } & $\mathrm{O} 1$ & $221.71 \pm 1.18$ & $180.8 \pm 1.66$ & $306.88 \pm 1.53$ & $229.5 \pm 1.13$ & $214.41 \pm 0.29$ & $230.66 \pm 3.03 \mathrm{ac}$ & \multirow{5}{*}{$\begin{array}{c}311.24 \pm \\
4.95 \mathrm{c}\end{array}$} \\
\hline & $\mathrm{O} 2$ & $282.94 \pm 0.81$ & $275.27 \pm 0.15$ & $297.53 \pm 0.99$ & $245.35 \pm 3.73$ & $263.3 \pm 0.66$ & $272.88 \pm 1.82 \mathrm{~cd}$ & \\
\hline & O3 & $303 \pm 0.48$ & $407.01 \pm 6.61$ & $312.31 \pm 0.65$ & $291.16 \pm 3.47$ & $334.74 \pm 0.85$ & $329.65 \pm 3.87 \mathrm{e}$ & \\
\hline & $\mathrm{O} 4$ & $361.04 \pm 0.46$ & $400.63 \pm 2.43$ & $506.98 \pm 1.93$ & $342.76 \pm 0.85$ & $447.57 \pm 0.86$ & $411.79 \pm 3.31 \mathrm{f}$ & \\
\hline & $\mathrm{O} 5$ & $167.66 \pm 1.13$ & $279.99 \pm 1.08$ & $197.36 \pm 1.76$ & $164.13 \pm 1.18$ & $179.44 \pm 0.56$ & $197.72 \pm 3.31 \mathrm{a}$ & \\
\hline \multirow{4}{*}{$\begin{array}{l}\text { 75\% orange/ } \\
25 \% \text { pumpkin }\end{array}$} & O6 & $270.37 \pm 3.8$ & $283.68 \pm 6.36$ & $292.68 \pm 2.86$ & $191.73 \pm 5.41$ & $189.83 \pm 1.91$ & $245.66 \pm 4.79 \mathrm{c}$ & \multirow{4}{*}{$\begin{array}{c}252.19 \pm \\
4.63 a\end{array}$} \\
\hline & O7 & $269.76 \pm 3.77$ & $288.35 \pm 4.41$ & $284.72 \pm 3.4$ & $227.34 \pm 6.12$ & $232.78 \pm 0.89$ & $260.59 \pm 3.81 \mathrm{~cd}$ & \\
\hline & O8 & $290.78 \pm 6.61$ & $312.57 \pm 2.13$ & $371.9 \pm 2.21$ & $261.28 \pm 5.35$ & $287.34 \pm 1.25$ & $304.78 \pm 4.01 \mathrm{~d}$ & \\
\hline & O9 & $170.17 \pm 3.04$ & $216.21 \pm 1.22$ & $206.8 \pm 2.76$ & $178.99 \pm 3.05$ & $170.41 \pm 0.45$ & $188.52 \pm 2.45 a$ & \\
\hline \multirow{3}{*}{$\begin{array}{l}75 \% \text { orange/ } \\
25 \% \text { papaya }\end{array}$} & $\mathrm{O} 10$ & $279.75 \pm 4.21$ & $299.91 \pm 1.83$ & $256.03 \pm 2.87$ & $224.45 \pm 2.81$ & $261.89 \pm 1.57$ & $264.41 \pm 2.87 \mathrm{~cd}$ & \multirow{3}{*}{$\begin{array}{l}284.18 \pm \\
5.82 \mathrm{~b}\end{array}$} \\
\hline & $\mathrm{O} 11$ & $291.96 \pm 2.54$ & $354.52 \pm 4.38$ & $224.66 \pm 1.45$ & $276.35 \pm 4.18$ & $327.02 \pm 2.07$ & $294.9 \pm 3.82 \mathrm{de}$ & \\
\hline & $\mathrm{O} 12$ & $383.6 \pm 2.46$ & $254.29 \pm 2$ & $424.21 \pm 1.23$ & $379.44 \pm 2.22$ & $502.83 \pm 6.5$ & $388.88 \pm 5.28 f$ & \\
\hline \multicolumn{2}{|c|}{ Mean of total storage period } & $274.4 \pm 4.59 \mathrm{AB}$ & $296.1 \pm 4.86 \mathrm{BC}$ & $306.84 \pm 5.31 \mathrm{C}$ & $251.04 \pm 4.94 \mathrm{~A}$ & $284.3 \pm 6.43 \mathrm{BC}$ & & \\
\hline \multicolumn{2}{|c|}{$\begin{array}{c}\text { Ratio of } \\
\text { (fructose:sweeteners) }\end{array}$} & $\begin{array}{l}\mathrm{O}(1,5,9) \\
(100 / 0)\end{array}$ & $\begin{array}{c}\mathrm{O}(2,6,10) \\
(75 / 25)\end{array}$ & $\begin{array}{l}\mathrm{O}(3,7,11) \\
(50 / 50)\end{array}$ & $\begin{array}{l}\mathrm{O}(4,8,12) \\
(25 / 75)\end{array}$ & & & \\
\hline \multicolumn{2}{|c|}{ Mean of sweetener formula } & $205.63 \pm 3.16 \mathrm{~A}$ & $260.98 \pm 3.34 \mathrm{~B}$ & $295.05 \pm 4.10 \mathrm{C}$ & $368.48 \pm 4.83 \mathrm{D}$ & & & \\
\hline \multirow{2}{*}{$\begin{array}{l}\text { LSD at } 0.05 \\
\text { for: }\end{array}$} & Storage (S) & Fruit (F) & Sweetener (T) & $\mathrm{S} * \mathrm{~F}$ & $\mathrm{~S} * \mathrm{~T}$ & $\mathrm{~F}^{*} \mathrm{~T}$ & $\mathrm{~S} * \mathrm{~F}^{*} \mathrm{~T}$ & \\
\hline & 23.96 & 21.43 & 21.43 & 41.51 & 47.93 & 37.12 & 83.01 & \\
\hline
\end{tabular}

a, b \& c: There is no significant difference $(\mathrm{P}>0.05)$ between any two means, within the same column have the same superscript letter. $n=3$. A, B \& $\mathrm{C}$ : There is no significant difference $(\mathrm{P}>0.05)$ between any two means for the same attribute within the same row have the same superscript letter.

whose indicated TPC decreasing during jam storage. Comparing between mean of sweetener formula the results indicated that TPC increased from $205.63 \pm 3.16 \mathrm{mg}$ GAE $100 \mathrm{~g}^{-1}$ in ratio of (100:0) to $368.48 \pm 4.83 \mathrm{mg}$ GAE $100 \mathrm{~g}^{-1}$ in ratio of (25:75). These results are in agreement with Shukla et al. [53] who remarked increases in TPC due to addition of stevioside in prepared diet jam. Moreover, Tadhani et al. [54] indicated that stevia plant containing $25.18 \mathrm{mg} \mathrm{gm}^{-1}$ TPC in stevia leaves.

Data in Table 4, indicated that mean of antioxidantactivity in jam types was ranged from $20.48 \pm 1.62$ to $25.47 \pm 1.63 \mu \mathrm{mol} \mathrm{TE} \mathrm{g}^{-1}$. These results indicated that there is a significant difference among all jam types. Increasing of antioxidant activity in formulas $\mathrm{O} 4, \mathrm{O} 8$ and $\mathrm{O} 12$ may be due to increasing of stevioside content. Shukla et al. [53] reported that stevia plant used as a good source of antioxidant. Tadhani et al. [54] reported that stevia is containing 9.66 to $38.24 \mathrm{mg}$ antioxidant. Comparing between mean of total storage period the results indicated that the highest antioxidant activity was $27.69 \pm 1.11 \mu \mathrm{mol} \mathrm{TE} \mathrm{g}^{-1}$ obtained at zero time while low antioxidant activity was $13.67 \pm 1.17 \mu \mathrm{mol} \mathrm{TE} \mathrm{g}^{-1}$ at the end of storage. This means that antioxidant activity decreased during storage for 12 month. There is a significant difference among all storage periods. These results were consistent with data reported previously [48] [49] [52] [55]-[59]. They demonstrated that the antioxidant activity of different berries significantly decreased after jam processing and storage period. This decrease can be attributed to the destruction of phytochemicals, phenolics compounds as well as L-ascorbic acid as a result of thermal treatment. Comparing between mean of sweetener formula the results indicated that highest antioxidant was $26.98 \pm 1.57 \mu \mathrm{mol} \mathrm{TE} \mathrm{g}^{-1}$ in ratio of (25:75) while low antioxidant activity was $17.32 \pm 1.19 \mu \mathrm{mol} \mathrm{TE} \mathrm{g}^{-1}$ in ratio of (100:0). These results may be due to addition of stevioside to diet jam which increasing antioxidant activity in prepared jams (Shukla et al. [53]).

The presented data in Table 5, illustrated the carotenoids content prepared jams. Carotenoids content was

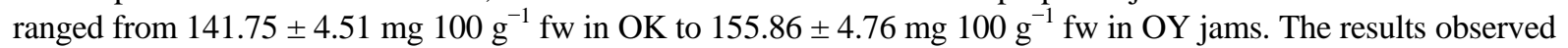


Table 4. Antioxidant activity ( $\mu \mathrm{mol} \mathrm{TE} \mathrm{g}^{-1} \mathrm{fw}$ ) of orange-based formulated low calories jams sweetened with fructose, stevioside and sucralose during 12 months of storage (mean $\pm \mathrm{SD})$.

\begin{tabular}{|c|c|c|c|c|c|c|c|c|}
\hline \multirow{2}{*}{ Fruit type } & \multirow{2}{*}{$\begin{array}{l}\text { Sweetener } \\
\text { formula }\end{array}$} & \multicolumn{5}{|c|}{ Storage period (month) } & \multirow{2}{*}{$\begin{array}{l}\text { Mean of storage } \\
\text { period }\end{array}$} & \multirow{2}{*}{$\begin{array}{l}\text { Mean of } \\
\text { fruit type }\end{array}$} \\
\hline & & 0 & 3 & 6 & 9 & 12 & & \\
\hline \multirow{5}{*}{ Orange } & $\mathrm{O} 1$ & $23.04 \pm 0.17$ & $24.29 \pm 0.08$ & $16.55 \pm 0.57$ & $16.81 \pm 0.61$ & $12.7 \pm 0.11$ & $18.68 \pm 1.09 \mathrm{~b}$ & \multirow{4}{*}{$\begin{array}{c}25.47 \pm \\
1.63 c\end{array}$} \\
\hline & $\mathrm{O} 2$ & $26.21 \pm 0.19$ & $26.08 \pm 0.17$ & $31.57 \pm 0.44$ & $18.22 \pm 0.45$ & $14.81 \pm 0.09$ & $23.38 \pm 1.32 \mathrm{~cd}$ & \\
\hline & $\mathrm{O} 3$ & $30.56 \pm 0.31$ & $30.54 \pm 0.13$ & $38.39 \pm 0.52$ & $20.49 \pm 0.35$ & $17.16 \pm 0.31$ & $27.43 \pm 1.54 \mathrm{e}$ & \\
\hline & $\mathrm{O} 4$ & $39.15 \pm 0.1$ & $41.01 \pm 0.16$ & $28.14 \pm 0.51$ & $28.32 \pm 0.47$ & $25.34 \pm 0.3$ & $32.39 \pm 1.19 f$ & \\
\hline & O5 & $24.59 \pm 0.12$ & $24.79 \pm 0.1$ & $16.83 \pm 0.43$ & $16.69 \pm 0.39$ & $10.74 \pm 0.19$ & $18.73 \pm 1.30 \mathrm{~b}$ & \multirow{5}{*}{$\begin{array}{c}22.66 \pm \\
1.57 \mathrm{~b}\end{array}$} \\
\hline \multirow{4}{*}{$\begin{array}{l}75 \% \text { orange/ } \\
25 \% \text { pumpkin }\end{array}$} & O6 & $26.42 \pm 0.22$ & $26.63 \pm 0.18$ & $31.75 \pm 0.34$ & $18.19 \pm 0.51$ & $10.66 \pm 0.1$ & $22.73 \pm 1.63 c$ & \\
\hline & $\mathrm{O} 7$ & $32.22 \pm 0.58$ & $27.53 \pm 0.4$ & $27.07 \pm 2$ & $19.13 \pm 0.36$ & $11.84 \pm 0.16$ & $23.56 \pm 1.77 \mathrm{~cd}$ & \\
\hline & $\mathrm{O} 8$ & $28.99 \pm 1.54$ & $25.48 \pm 1.06$ & $29.4 \pm 0.58$ & $27.84 \pm 0.53$ & $16.39 \pm 0.11$ & $25.62 \pm 1.27 d$ & \\
\hline & O9 & $17.63 \pm 0.3$ & $16.65 \pm 0.82$ & $14.51 \pm 0.29$ & $14.7 \pm 0.23$ & $9.35 \pm 0.03$ & $14.57 \pm 0.86 a$ & \\
\hline \multirow{3}{*}{$\begin{array}{l}75 \% \text { orange/ } \\
25 \% \text { papaya }\end{array}$} & $\mathrm{O} 10$ & $24.28 \pm 0.15$ & $22.65 \pm 0.5$ & $27.26 \pm 0.19$ & $16.13 \pm 0.34$ & $11.04 \pm 0.09$ & $20.27 \pm 1.38 b$ & \multirow{3}{*}{$\begin{array}{c}20.48 \pm \\
1.62 \mathrm{a}\end{array}$} \\
\hline & $\mathrm{O} 11$ & $26.64 \pm 0.15$ & $28.27 \pm 0.1$ & $32.23 \pm 0.03$ & $20.07 \pm 0.58$ & $13.59 \pm 0.16$ & $24.16 \pm 1.40 \mathrm{~cd}$ & \\
\hline & $\mathrm{O} 12$ & $32.53 \pm 0.44$ & $30.28 \pm 0.25$ & $20.57 \pm 0.69$ & $20.8 \pm 0.75$ & $10.41 \pm 0.24$ & $22.92 \pm 1.77 c$ & \\
\hline \multicolumn{2}{|c|}{ Mean of total storage period } & $27.69 \pm 1.11 \mathrm{E}$ & $27.02 \pm 1.13 \mathrm{D}$ & $26.19 \pm 1.54 \mathrm{C}$ & $19.78 \pm 1.02 \mathrm{~B}$ & \multirow[t]{2}{*}{$13.67 \pm 1.17 \mathrm{~A}$} & & \\
\hline \multicolumn{2}{|c|}{$\begin{array}{c}\text { Ratio of } \\
\text { (fructose:sweeteners) }\end{array}$} & $\begin{array}{c}\mathrm{O}(1,5,9) \\
(100 / 0)\end{array}$ & $\begin{array}{c}\mathrm{O}(2,6,10) \\
(75 / 25)\end{array}$ & $\begin{array}{c}\mathrm{O}(3,7,11) \\
(50 / 50)\end{array}$ & $\begin{array}{c}\mathrm{O}(4,8,12) \\
(25 / 75)\end{array}$ & & & \\
\hline \multicolumn{2}{|c|}{ Mean of sweetener formula } & $17.32 \pm 1.19 \mathrm{~A}$ & $22.13 \pm 1.45 \mathrm{~B}$ & \multicolumn{3}{|c|}{$25.05 \pm 1.58 \mathrm{C} 26.98 \pm 1.57 \mathrm{D}$} & & \\
\hline \multirow{2}{*}{ LSD at 0.05 for: } & Storage (S) & Fruit (F) & Sweetener ( $\mathrm{T}$ ) & $\mathrm{S} * \mathrm{~F}$ & $\mathrm{~S} * \mathrm{~T}$ & $\mathrm{~F}^{*} \mathrm{~T}$ & $\mathrm{~S} * \mathrm{~F}^{*} \mathrm{~T}$ & \\
\hline & 1.16 & 1.04 & 1.04 & 2.02 & 2.33 & 1.80 & 4.03 & \\
\hline
\end{tabular}

a, b \& c: There is no significant difference $(\mathrm{P}>0.05)$ between any two means, within the same column have the same superscript letter. $n=3$. A, B \& $\mathrm{C}$ : There is no significant difference $(\mathrm{P}>0.05)$ between any two means for the same attribute within the same row have the same superscript letter.

Table 5. Carotenoids content (mg $100 \mathrm{~g}^{-1} \mathrm{fw}$ ) of orange-based formulated low calories jams sweetened with fructose, stevioside and sucralose during 12 months of storage (mean $\pm \mathrm{SD}$ ).

\begin{tabular}{|c|c|c|c|c|c|c|c|c|}
\hline \multirow{2}{*}{ Fruit type } & \multirow{2}{*}{$\begin{array}{l}\text { Sweetener } \\
\text { formula }\end{array}$} & \multicolumn{5}{|c|}{ Storage period (month) } & \multirow{2}{*}{$\begin{array}{c}\text { Mean of storage } \\
\text { period }\end{array}$} & \multirow{2}{*}{$\begin{array}{l}\text { Mean of } \\
\text { fruit type }\end{array}$} \\
\hline & & 0 & 3 & 6 & 9 & 12 & & \\
\hline \multirow{4}{*}{ Orange } & $\mathrm{O} 1$ & $127.78 \pm 2.9$ & $127.69 \pm 2.92$ & $118.23 \pm 2.32$ & $177.95 \pm 5.85$ & $115.03 \pm 0.94$ & $133.34 \pm 3.72 \mathrm{abc}$ & \multirow{4}{*}{$\begin{array}{l}153.22 \pm \\
5.80 \mathrm{a}\end{array}$} \\
\hline & $\mathrm{O} 2$ & $110.4 \pm 2.62$ & $105.73 \pm 2.58$ & $101.36 \pm 2.6$ & $130.42 \pm 6.52$ & $163.01 \pm 3.08$ & $122.19 \pm 3.91 \mathrm{a}$ & \\
\hline & $\mathrm{O} 3$ & $153.55 \pm 2.25$ & $124.69 \pm 1.07$ & $139.62 \pm 1.25$ & $263.51 \pm 2.48$ & $173.54 \pm 2.74$ & $170.98 \pm 4.29$ cde & \\
\hline & $\mathrm{O} 4$ & $157.52 \pm 5.01$ & $130.37 \pm 1.65$ & $179.94 \pm 7.87$ & $97.43 \pm 3.43$ & $366.61 \pm 0.78$ & $186.38 \pm 8.00$ de & \\
\hline \multirow{4}{*}{$\begin{array}{l}75 \% \text { orange/ } \\
25 \% \text { pumpkin }\end{array}$} & O5 & $135.4 \pm 4.97$ & $87.38 \pm 3.67$ & $157.57 \pm 7.73$ & $76.22 \pm 8.19$ & $119.42 \pm 0.33$ & $115.20 \pm 5.66 a$ & \multirow{5}{*}{$\begin{array}{c}141.75 \pm \\
4.51 \mathrm{a}\end{array}$} \\
\hline & O6 & $131.48 \pm 1.67$ & $144.86 \pm 0.22$ & $161.1 \pm 2.3$ & $94.96 \pm 6.62$ & $122.96 \pm 1.54$ & $131.07 \pm 3.20 \mathrm{abc}$ & \\
\hline & O7 & $127.82 \pm 1.44$ & $175.19 \pm 2.7$ & $139.67 \pm 0.88$ & $176.33 \pm 3.44$ & $136.38 \pm 3.09$ & $151.08 \pm 2.78 \mathrm{abcd}$ & \\
\hline & O8 & $123.29 \pm 1.71$ & $130.87 \pm 1.47$ & $149.7 \pm 3.19$ & $255.69 \pm 5.9$ & $188.7 \pm 1.37$ & $169.65 \pm 4.95$ bcde & \\
\hline \multirow{3}{*}{$\begin{array}{l}75 \% \text { orange/ } \\
25 \% \text { papaya }\end{array}$} & O9 & $114.16 \pm 1.38$ & $112.9 \pm 1.35$ & $141.69 \pm 1.67$ & $111.52 \pm 1.77$ & $157.26 \pm 0.81$ & $127.51 \pm 2.08 \mathrm{ab}$ & \\
\hline & $\mathrm{O} 10$ & $93.61 \pm 1.82$ & $136.8 \pm 1.41$ & $112.16 \pm 0.98$ & $96.48 \pm 9.13$ & $237.83 \pm 3.09$ & $135.37 \pm 5.85 a b c$ & \multirow{3}{*}{$\begin{array}{c}155.86 \pm \\
4.76 \mathrm{a}\end{array}$} \\
\hline & $\mathrm{O} 11$ & $143.03 \pm 3.17$ & $157.69 \pm 1.56$ & $104.56 \pm 1.01$ & $175.35 \pm 5.54$ & $200.56 \pm 1.72$ & $156.24 \pm 3.79 \mathrm{abcd}$ & \\
\hline \multirow{3}{*}{\multicolumn{2}{|c|}{$\begin{array}{c}\text { Mean of total storage period } \\
\text { Ratio of } \\
\text { (fructose:sweeteners) }\end{array}$}} & $210.06 \pm 4.55$ & $208.72 \pm 4.62$ & $204.74 \pm 2.81$ & $189.32 \pm 8.4$ & $208.79 \pm 2.34$ & \multirow[t]{2}{*}{$204.33 \pm 4.21 \mathrm{e}$} & \\
\hline & & $135.68 \pm 3.65 \mathrm{~A}$ & $136.91 \pm 3.39 \mathrm{~A}$ & $142.53 \pm 4.14 \mathrm{~A}$ & $153.77 \pm 6.87 \mathrm{~A}$ & $182.51 \pm 5.29 \mathrm{~B}$ & & \\
\hline & & $\mathrm{O}(1,5,9)(100 / 0)$ & $\begin{array}{c}\mathrm{O}(2,6,10) \\
(75 / 25)\end{array}$ & $\begin{array}{c}\mathrm{O}(3,7,11) \\
(50 / 50)\end{array}$ & $\begin{array}{l}\mathrm{O}(4,8,12) \\
(25 / 75)\end{array}$ & & & \\
\hline \multicolumn{2}{|c|}{ Mean of sweetener formula } & $125.35 \pm 3.99 \mathrm{~A}$ & $129.54 \pm 4.42 \mathrm{~A}$ & $159.43 \pm 3.68 B$ & $186.78 \pm 5.89 \mathrm{C}$ & & & \\
\hline \multirow{2}{*}{$\begin{array}{l}\text { LSD at } \\
0.05 \text { for: }\end{array}$} & Storage (S) & Fruit $(F)$ & Sweetener ( $\mathrm{T}$ ) & $\mathrm{S} * \mathrm{~F}$ & $\mathrm{~S} * \mathrm{~T}$ & $\mathrm{~F}^{*} \mathrm{~T}$ & $\mathrm{~S} * \mathrm{~F}^{*} \mathrm{~T}$ & \\
\hline & 21.12 & 18.89 & 18.89 & 36.58 & 42.24 & 32.72 & 73.17 & \\
\hline
\end{tabular}

a, b \& c: There is no significant difference $(\mathrm{P}>0.05)$ between any two means, within the same column have the same superscript letter. $n=3$. A, B \& $\mathrm{C}$ : There is no significant difference $(\mathrm{P}>0.05)$ between any two means for the same attribute within the same row have the same superscript letter. 
that there is no significant difference among all of jam types. These results are in agreement with [28] [60]. Comparing between mean of total storage period the results indicated that higher carotenoids content was $182.51 \pm$ $5.29 \mathrm{mg} 100 \mathrm{~g} \mathrm{~g}^{-1} \mathrm{fw}$ at the end of storage period while lower carotenoids content was $135.68 \pm 3.65 \mathrm{mg} 100 \mathrm{~g}^{-1}$ $\mathrm{fw}$ at zero time. These results indicated that carotenoids may be released from the matrix during storage. These results are in agreement with Correa et al. [49] who observed that carotenoids increased from $36.66 \pm 1.17$ to $62.18 \pm 1.48 \mathrm{mg} 100 \mathrm{~g}^{-1} \mathrm{fw}$ at day-1and day-90 in grape juice, respectively. Comparing between mean of swee-

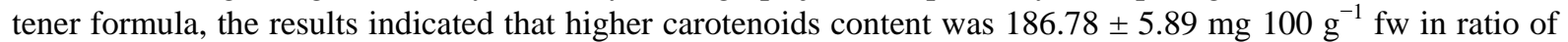

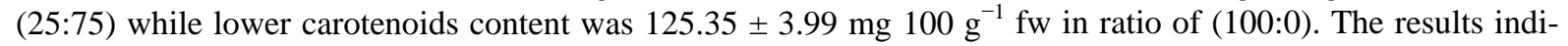
cated that there is no significant difference between the ratios (100:0) and (75:25) while there is a significant difference between (50:50) and (25:75) ratios.

\subsection{Vitamin C and Total Soluble Solids Contents}

Vitamin C is involved in protein metabolism, collagen synthesis and an important physiological antioxidant [61]. In Table 6, results indicated that there is a significant difference between jam types. The results demonstrated

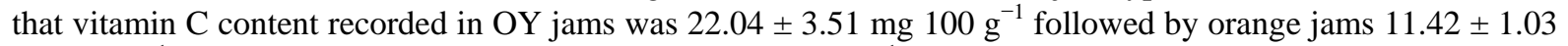
$\mathrm{mg} 100 \mathrm{~g}^{-1} \mathrm{fw}$ while OK jams recorded $9.59 \pm 1.03 \mathrm{mg} 100 \mathrm{~g}^{-1} \mathrm{fw}$. The difference of vitamin C content in formulas may be due to the difference of vitamin content initial fruits pulp. Johnson et al. [62] reported that vitamine C content in pumpkin pulp $15.25 \mathrm{mg} 100 \mathrm{~g}^{-1}$ while Nwofia et al. [63] reported that vitamin C content in papaya pulp ranged from 36.37 to $43.41 \mathrm{mg} 100 \mathrm{~g} \mathrm{~g}^{-1}$. Comparing between mean of total storage period the results indicated that maximum vitamin $\mathrm{C}$ content was $21.21 \pm 2.96 \mathrm{mg} 100 \mathrm{~g}^{-1}$ at zero time while deceased to $6.01 \pm$ $0.50 \mathrm{mg} 100 \mathrm{~g}^{-1}$ at 12 month storage. These results are in agreement with [50] [64] reported that vitamin C decreased in low calorie jam during storage. The loss of vitamin C in the initial stages is because of the presence of

Table 6. Vitamin C (mg $100 \mathrm{~g}^{-1} \mathrm{fw}$ ) content of orange-based formulated low calories jams sweetened with fructose, stevioside and sucralose during 12 months of storage (mean $\pm \mathrm{SD}$ ).

\begin{tabular}{|c|c|c|c|c|c|c|c|c|}
\hline \multirow{2}{*}{ Fruit type } & \multirow{2}{*}{$\begin{array}{l}\text { Sweetener } \\
\text { formula }\end{array}$} & \multicolumn{5}{|c|}{ Storage period (month) } & \multirow{2}{*}{$\begin{array}{l}\text { Mean of storage } \\
\text { period }\end{array}$} & \multirow{2}{*}{$\begin{array}{l}\text { Mean of } \\
\text { fruit typ }\end{array}$} \\
\hline & & 0 & 3 & 6 & 9 & 12 & & \\
\hline \multirow{4}{*}{ Orange } & $\mathrm{O} 1$ & $14.91 \pm 0.06$ & $14.65 \pm 0.17$ & $9.71 \pm 0.92$ & $11.27 \pm 0.39$ & $6.93 \pm 0.3$ & $11.49 \pm 0.99 b c$ & \multirow{4}{*}{$\begin{array}{l}11.42 \pm \\
1.03 \mathrm{~b}\end{array}$} \\
\hline & $\mathrm{O} 2$ & $14.35 \pm 0.16$ & $14.56 \pm 0.14$ & $12.48 \pm 0.44$ & $10.75 \pm 0.24$ & $4.85 \pm 0.36$ & $11.40 \pm 1.11 \mathrm{bc}$ & \\
\hline & $\mathrm{O} 3$ & $16.55 \pm 0.26$ & $16.12 \pm 0.34$ & $11.79 \pm 0.32$ & $11.96 \pm 0.3$ & $5.2 \pm 0.46$ & $12.32 \pm 1.23 \mathrm{c}$ & \\
\hline & $\mathrm{O} 4$ & $11.37 \pm 0.14$ & $11.22 \pm 0.26$ & $11.96 \pm 0.3$ & $11.27 \pm 0.39$ & $6.41 \pm 0.12$ & $10.44 \pm 0.69 b$ & \\
\hline \multirow{4}{*}{$\begin{array}{l}75 \% \text { orange/ } \\
25 \% \text { pumpkin }\end{array}$} & O5 & $11.35 \pm 0.06$ & $10.93 \pm 0.11$ & $7.63 \pm 0.47$ & $7.63 \pm 0.47$ & $7.45 \pm 0.48$ & $9.00 \pm 0.67 \mathrm{a}$ & \multirow{4}{*}{$\begin{array}{l}9.59 \pm \\
1.03 a\end{array}$} \\
\hline & O6 & $13.26 \pm 0.36$ & $13.35 \pm 0.36$ & $10.4 \pm 0.43$ & $9.19 \pm 0.6$ & $5.03 \pm 0.35$ & $10.24 \pm 1.06 \mathrm{ab}$ & \\
\hline & $\mathrm{O} 7$ & $14.04 \pm 0.42$ & $14.65 \pm 0.49$ & $8.49 \pm 0.27$ & $8.84 \pm 0.35$ & $4.85 \pm 0.36$ & $10.17 \pm 1.24 \mathrm{ab}$ & \\
\hline & $\mathrm{O} 8$ & $13.15 \pm 0.18$ & $11.61 \pm 0.25$ & $7.97 \pm 0.46$ & $5.2 \pm 0.23$ & $6.76 \pm 0.4$ & $8.94 \pm 1.07 a$ & \\
\hline \multirow{4}{*}{$\begin{array}{l}75 \% \text { orange/ } \\
25 \% \text { papaya }\end{array}$} & O9 & $20.19 \pm 0.16$ & $19.87 \pm 0.12$ & $17.68 \pm 0.33$ & $6.76 \pm 0.2$ & $6.59 \pm 0.31$ & $14.22 \pm 1.72 \mathrm{~d}$ & \multirow{4}{*}{$\begin{array}{c}22.04 \pm \\
3.51 \mathrm{c}\end{array}$} \\
\hline & $\mathrm{O} 10$ & $29.21 \pm 0.25$ & $28.51 \pm 0.37$ & $15.43 \pm 0.2$ & $10.4 \pm 0.16$ & $5.37 \pm 0.34$ & $17.78 \pm 2.37 \mathrm{e}$ & \\
\hline & O11 & $57.37 \pm 0.1$ & $56.33 \pm 0.28$ & $13.17 \pm 0.3$ & $11.79 \pm 0.23$ & $5.2 \pm 0.23$ & $28.77 \pm 4.46 f$ & \\
\hline & $\mathrm{O} 12$ & $38.75 \pm 0.13$ & $36.55 \pm 0.25$ & $44.03 \pm 1.2$ & $10.05 \pm 0.25$ & $7.45 \pm 0.29$ & $27.37 \pm 3.11 \mathrm{~g}$ & \\
\hline \multicolumn{2}{|c|}{ Mean of total storage period } & $21.21 \pm 2.96 \mathrm{D}$ & $20.70 \pm 2.91 \mathrm{D}$ & $14.23 \pm 2.60 \mathrm{C}$ & $9.59 \pm 0.73 B$ & $6.01 \pm 0.50 \mathrm{~A}$ & & \\
\hline \multicolumn{2}{|c|}{$\begin{array}{c}\text { Ratio of } \\
\text { (fructose:sweeteners) }\end{array}$} & $\begin{array}{l}\mathrm{O}(1,5,9) \\
(100 / 0)\end{array}$ & $\begin{array}{l}\mathrm{O}(2,6,10) \\
(75 / 25)\end{array}$ & $\begin{array}{c}\mathrm{O}(3,7,11) \\
(50 / 50)\end{array}$ & $\begin{array}{c}\mathrm{O}(4,8,12) \\
(25 / 75)\end{array}$ & & & \\
\hline \multicolumn{2}{|c|}{ Mean of sweetener formula } & $11.57 \pm 1.41 \mathrm{~A}$ & $13.14 \pm 1.97 \mathrm{~B}$ & $17.09 \pm 3.93 \mathrm{D}$ & $15.58 \pm 3.21 \mathrm{C}$ & & & \\
\hline \multirow{2}{*}{ LSD at 0.05 for: } & Storage (S) & Fruit (F) & Sweetener (T) & $\mathrm{S}^{*} \mathrm{~F}$ & $\mathrm{~S} * \mathrm{~T}$ & $\mathrm{~F}^{*} \mathrm{~T}$ & $\mathrm{~S} * \mathrm{~F} * \mathrm{~T}$ & \\
\hline & 0.70 & 0.63 & 0.63 & 1.21 & 1.40 & 1.09 & 2.43 & \\
\hline
\end{tabular}

a, b \& c: There is no significant difference $(\mathrm{P}>0.05)$ between any two means, within the same column have the same superscript letter. $n=3$. A, B \& $\mathrm{C}$ : There is no significant difference $(\mathrm{P}>0.05)$ between any two means for the same attribute within the same row have the same superscript letter. 
oxygen in the headspace or this could due to oxidation or degradation of the thermolabile ascorbic acid into dehydrate ascorbic acid upon storage [64]. Comparing between mean of sweetener formula the results indicated

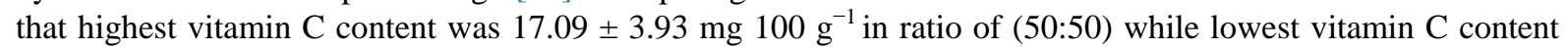

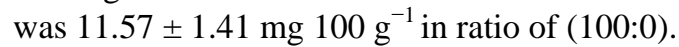

The mean of TSS was ranged from $31.98 \pm 1.15$ to $34.13 \pm 1.19$ [Brix] in OK and Orange jams, respectively (Table 7). Significant difference between OK and (orange and OY) jams was found. Comparing between mean of storage period the results indicated that TSS ranged from a low of $22.53 \pm 0.05$ in O8 to a high of $43.37 \pm$ 0.13 in O1. Comparing between mean of total storage period the results indicated that TSS was almost stable and no trend could be observed even a slight increases was recorded at the end of storage period. These results are in agreement with the results of [65]-[67] who observed minor increase in TSS of strawberry and watermelon lemon jams during storage. The increase in TSS contents of the product may be due to acid hydrolysis of polysaccharides especially gums and pectin into simple sugars [68]. Comparing between mean of sweetener formula a logical significant differences was observed with increasing the replacement level of fructose.

\subsection{Organoleptical Parameters of Orange-Based Jams}

Twelve low calorie orange-based formulated jams were prepared mainly from orange, pumpkin and papaya then sweetened using F, St and Su as mentioned in materials and methods in Table 1, data were illustrated in Tables 8-12. Color, taste, odor, texture, and bitterness of prepared jams were organoleptically evaluated. The effect of fructose replacement by (St \& Su) on sensory attributes was investigated as well.

The obtained data in Table 8 indicated that no significant difference $(\mathrm{P}<0.05)$ was found in color scores between prepared OK and OY or orange jams. These findings are in agreements with [69]. Also Abdullah et al.

Table 7. Total soluble solids (Brix) of orange-based formulated low calories jams sweetened with fructose, stevioside and sucralose during 12 months of storage (mean $\pm \mathrm{SD}$ ).

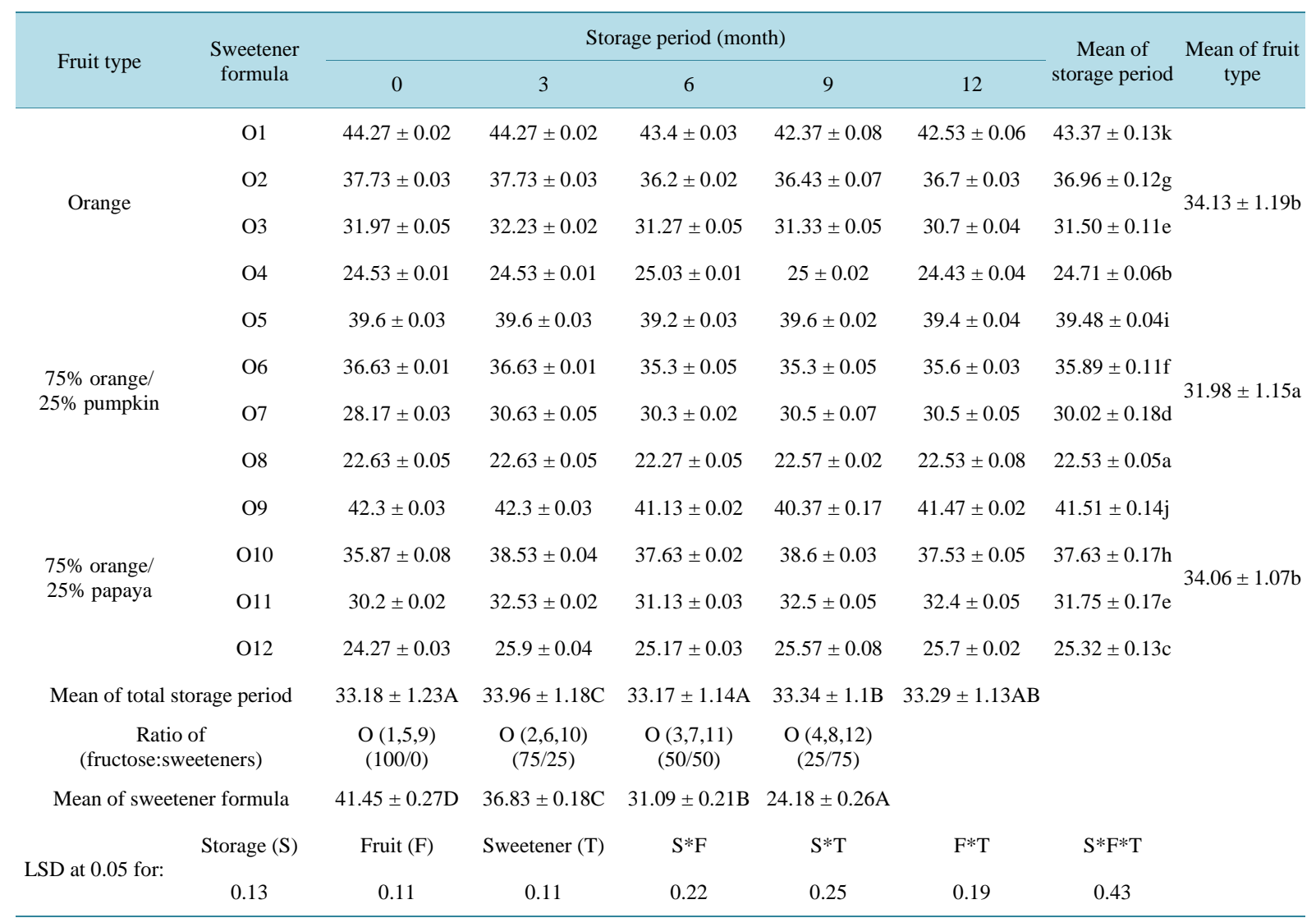

a, b \& c: There is no significant difference $(\mathrm{P}>0.05)$ between any two means, within the same column have the same superscript letter. $n=3$. A, B \& $\mathrm{C}$ : There is no significant difference $(\mathrm{P}>0.05)$ between any two means for the same attribute within the same row have the same superscript letter. 
Table 8. Color of orange-based formulated low calories jams sweetened with fructose, stevioside and sucralose during 12 months of storage (mean $\pm \mathrm{SD}$ ).

\begin{tabular}{|c|c|c|c|c|c|c|c|c|}
\hline \multirow{2}{*}{ Fruit type } & \multirow{2}{*}{$\begin{array}{l}\text { Sweetener } \\
\text { formula }\end{array}$} & \multicolumn{5}{|c|}{ Storage period (month) } & \multirow{2}{*}{$\begin{array}{l}\text { Mean of storage } \\
\text { period }\end{array}$} & \multirow{2}{*}{$\begin{array}{l}\text { Mean of } \\
\text { fruit type }\end{array}$} \\
\hline & & 0 & 3 & 6 & 9 & 12 & & \\
\hline \multirow{5}{*}{ Orange } & $\mathrm{O} 1$ & $5.08 \pm 0.89$ & $5 \pm 1.11$ & $2.3 \pm 1.2$ & $2.6 \pm 1.22$ & $2.55 \pm 1.13$ & $3.51 \pm 1.67 \mathrm{bO}$ & \multirow{4}{*}{$\begin{array}{c}3.45 \pm \\
1.53 \mathrm{a}\end{array}$} \\
\hline & $\mathrm{O} 2$ & $5.1 \pm 0.63$ & $4.3 \pm 1.11$ & $3.1 \pm 1.06$ & $2.03 \pm 0.64$ & $2.08 \pm 0.67$ & $3.35 \pm 1.48 b$ & \\
\hline & $\mathrm{O} 3$ & $4.7 \pm 0.72$ & $4.6 \pm 1.37$ & $3 \pm 1.2$ & $2.1 \pm 0.84$ & $2.05 \pm 0.75$ & $3.29 \pm 1.54 b$ & \\
\hline & $\mathrm{O} 4$ & $4.85 \pm 1.16$ & $4.9 \pm 0.96$ & $3 \pm 1.2$ & $2.8 \pm 0.88$ & $2.75 \pm 0.84$ & $3.65 \pm 1.42 \mathrm{Od}$ & \\
\hline & O5 & $5.35 \pm 1.25$ & $5.2 \pm 0.99$ & $2.8 \pm 1.09$ & $2.9 \pm 1.15$ & $2.9 \pm 1.1$ & $3.83 \pm 1.62 \mathrm{df}$ & \multirow{5}{*}{$\begin{array}{l}3.81 \pm \\
1.57 \mathrm{~b}\end{array}$} \\
\hline \multirow{4}{*}{$\begin{array}{l}75 \% \text { orange/ } \\
25 \% \text { pumpkin }\end{array}$} & O6 & $5.41 \pm 0.79$ & $4.6 \pm 1.3$ & $2.9 \pm 1.06$ & $2.6 \pm 1.03$ & $2.6 \pm 0.98$ & $3.61 \pm 1.56 \mathrm{O}$ & \\
\hline & O7 & $5.58 \pm 0.87$ & $5.2 \pm 1.09$ & $3.2 \pm 0.99$ & $2.8 \pm 1.09$ & $2.78 \pm 1.05$ & $3.91 \pm 1.59 f$ & \\
\hline & O8 & $5.28 \pm 0.99$ & $5 \pm 1.01$ & $3.6 \pm 1.3$ & $2.8 \pm 1.09$ & $2.8 \pm 1.07$ & $3.90 \pm 1.52 f$ & \\
\hline & O9 & $4.68 \pm 1.14$ & $3.7 \pm 1.11$ & $2.5 \pm 1.22$ & $2.1 \pm 1.15$ & $2.13 \pm 1.14$ & $3.02 \pm 1.53 a$ & \\
\hline \multirow{3}{*}{$\begin{array}{l}75 \% \text { orange/ } \\
25 \% \text { papaya }\end{array}$} & $\mathrm{O} 10$ & $4.98 \pm 1.07$ & $4.2 \pm 1.09$ & $2.8 \pm 0.88$ & $2.4 \pm 1.03$ & $2.38 \pm 1.03$ & $3.35 \pm 1.46 b$ & \multirow{3}{*}{$\begin{array}{c}3.56 \pm \\
1.53 a\end{array}$} \\
\hline & $\mathrm{O} 11$ & $5.18 \pm 1.15$ & $5 \pm 1.28$ & $3.5 \pm 1.3$ & $3 \pm 1.28$ & $2.98 \pm 1.27$ & $3.93 \pm 1.58 \mathrm{f}$ & \\
\hline & $\mathrm{O} 12$ & $4.65 \pm 1.21$ & $4.5 \pm 1.52$ & $3.9 \pm 1.39$ & $3.3 \pm 0.91$ & $3.25 \pm 0.95$ & $3.92 \pm 1.34 \mathrm{f}$ & \\
\hline \multicolumn{2}{|c|}{ Mean of total storage period } & $5.07 \pm 1.04 \mathrm{D}$ & $4.68 \pm 1.24 \mathrm{O}$ & $3.05 \pm 1.23 \mathrm{~B}$ & $2.62 \pm 1.10 \mathrm{~A}$ & $2.6 \pm 1.07 \mathrm{~A}$ & & \\
\hline \multicolumn{2}{|c|}{$\begin{array}{c}\text { Ratio of } \\
\text { (fructose:sweeteners) }\end{array}$} & $\begin{array}{c}\mathrm{O}(1,5,9) \\
(100 / 0)\end{array}$ & $\begin{array}{c}\mathrm{O}(2,6,10) \\
(75 / 25)\end{array}$ & $\begin{array}{c}\mathrm{O}(3,7,11) \\
(50 / 50)\end{array}$ & $\begin{array}{c}\mathrm{O}(4,8,12) \\
(25 / 75)\end{array}$ & & & \\
\hline \multicolumn{2}{|c|}{ Mean of sweetener formula } & $3.45 \pm 1.64 \mathrm{~A}$ & $3.44 \pm 1.5 \mathrm{~A}$ & $3.71 \pm 1.59 \mathrm{~B}$ & $3.82 \pm 1.43 \mathrm{~B}$ & & & \\
\hline \multirow{2}{*}{ LSD at 0.05 for: } & Storage (S) & Fruit (F) & Sweetener (T) & $\mathrm{S} * \mathrm{~F}$ & $\mathrm{~S} * \mathrm{~T}$ & $\mathrm{~F}^{*} \mathrm{~T}$ & $\mathrm{~S} * \mathrm{~F}^{*} \mathrm{~T}$ & \\
\hline & 0.14 & 0.12 & 0.11 & 0.24 & 0.27 & 0.21 & 0.47 & \\
\hline
\end{tabular}

a, b \& O: There is no significant difference $(\mathrm{P}>0.05)$ between any two means, within the same column have the same superscript letter. $n=40$. A, B \& O: There is no significant difference $(\mathrm{P}>0.05)$ between any two means for the same attribute within the same row have the same superscript letter.

Table 9. Taste of orange-based formulated low calories jams sweetened with fructose, stevioside and sucralose during 12 months of storage (mean $\pm \mathrm{SD}$ ).

\begin{tabular}{|c|c|c|c|c|c|c|c|c|}
\hline \multirow{2}{*}{ Fruit type } & \multirow{2}{*}{$\begin{array}{l}\text { Sweetener } \\
\text { formula }\end{array}$} & \multicolumn{5}{|c|}{ Storage period (month) } & \multirow{2}{*}{$\begin{array}{c}\text { Mean of storage } \\
\text { period }\end{array}$} & \multirow{2}{*}{$\begin{array}{c}\text { Mean of fruit } \\
\text { type }\end{array}$} \\
\hline & & 0 & 3 & 6 & 9 & 12 & & \\
\hline \multirow{5}{*}{ Orange } & $\mathrm{O} 1$ & $4.9 \pm 1.03$ & $4.7 \pm 1.11$ & $4.1 \pm 0.71$ & $2.9 \pm 0.96$ & $2.93 \pm 0.94$ & $3.91 \pm 1.28 f g h$ & \multirow{5}{*}{$3.76 \pm 1.29 b$} \\
\hline & $\mathrm{O} 2$ & $5.4 \pm 0.84$ & $4.6 \pm 1.58$ & $4.1 \pm 0.84$ & $2.89 \pm 0.69$ & $2.95 \pm 0.66$ & $4.01 \pm 1.37 \mathrm{~h}$ & \\
\hline & $\mathrm{O} 3$ & $4.9 \pm 0.87$ & $4.5 \pm 1.13$ & $3.9 \pm 0.55$ & $2.6 \pm 0.67$ & $2.65 \pm 0.7$ & $3.71 \pm 1.24$ Odf & \\
\hline & $\mathrm{O} 4$ & $4.33 \pm 1.03$ & $3.9 \pm 1.46$ & $3.6 \pm 0.93$ & $2.6 \pm 0.67$ & $2.63 \pm 0.67$ & $3.41 \pm 1.21 \mathrm{ab}$ & \\
\hline & $\mathrm{O} 5$ & $4.78 \pm 1.07$ & $5.1 \pm 1.32$ & $3.4 \pm 1.37$ & $3.2 \pm 0.76$ & $3.23 \pm 0.7$ & $3.94 \pm 1.35 \mathrm{gh}$ & \\
\hline \multirow{4}{*}{$\begin{array}{l}75 \% \text { orange/ } \\
25 \% \text { pumpkin }\end{array}$} & O6 & $5.21 \pm 0.57$ & $4.2 \pm 1.26$ & $3.8 \pm 0.88$ & $2.8 \pm 0.99$ & $2.85 \pm 0.98$ & $3.76 \pm 1.31 \mathrm{dfg}$ & \multirow{4}{*}{$3.86 \pm 1.35 c$} \\
\hline & $\mathrm{O} 7$ & $5.55 \pm 1.04$ & $4.6 \pm 1.13$ & $3.6 \pm 0.81$ & $3.2 \pm 0.76$ & $3.15 \pm 0.77$ & $4.02 \pm 1.30 \mathrm{~h}$ & \\
\hline & O8 & $4.75 \pm 1.78$ & $4.6 \pm 1.37$ & $3.7 \pm 1.11$ & $2.8 \pm 0.61$ & $2.8 \pm 0.61$ & $3.73 \pm 1.44 \mathrm{df}$ & \\
\hline & O9 & $4.34 \pm 1.17$ & $4 \pm 1.11$ & $3.2 \pm 1.09$ & $2.6 \pm 1.03$ & $2.65 \pm 1$ & $3.35 \pm 1.28 \mathrm{a}$ & \\
\hline \multirow{3}{*}{$\begin{array}{l}75 \% \text { orange/ } \\
25 \% \text { papaya }\end{array}$} & $\mathrm{O} 10$ & $4.38 \pm 1.29$ & $4.1 \pm 1.66$ & $3.9 \pm 0.84$ & $2.7 \pm 0.79$ & $2.78 \pm 0.73$ & $3.57 \pm 1.31 \mathrm{bOd}$ & \multirow{3}{*}{$3.54 \pm 1.26 a$} \\
\hline & $\mathrm{O} 11$ & $4.63 \pm 1.25$ & $4.2 \pm 1.34$ & $3.6 \pm 0.81$ & $3.1 \pm 1.15$ & $3.1 \pm 1.08$ & $3.73 \pm 1.28 \mathrm{df}$ & \\
\hline & $\mathrm{O} 12$ & $3.58 \pm 1.2$ & $4.4 \pm 1.13$ & $3.6 \pm 0.67$ & $3 \pm 1.01$ & $3 \pm 0.96$ & $3.52 \pm 1.13 \mathrm{abO}$ & \\
\hline \multirow{2}{*}{\multicolumn{2}{|c|}{$\begin{array}{c}\text { Mean of total storage period } \\
\text { Ratio of } \\
\text { (fructose:sweeteners) }\end{array}$}} & $4.73 \pm 1.23 \mathrm{D}$ & $4.41 \pm 1.34 \mathrm{O}$ & $3.71 \pm 0.94 \mathrm{~B}$ & $2.87 \pm 0.88 \mathrm{~A}$ & $2.89 \pm 0.84 \mathrm{~A}$ & & \\
\hline & & $\begin{array}{l}\mathrm{O}(1,5,9) \\
(100 / 0)\end{array}$ & $\begin{array}{c}\mathrm{O}(2,6,10) \\
(75 / 25)\end{array}$ & $\begin{array}{l}\mathrm{O}(3,7,11) \\
(50 / 50)\end{array}$ & $\begin{array}{l}\mathrm{O}(4,8,12) \\
(25 / 75)\end{array}$ & & & \\
\hline \multicolumn{2}{|c|}{ Mean of sweetener formula } & $3.73 \pm 1.33 \mathrm{~B}$ & $3.78 \pm 1.34 \mathrm{~B}$ & $3.82 \pm 1.28 \mathrm{~B}$ & $3.55 \pm 1.27 \mathrm{~A}$ & & & \\
\hline \multirow{2}{*}{ LSD at 0.05 for: } & Storage (S) & Fruit (F) & Sweetener (T) & $\mathrm{S}^{*} \mathrm{~F}$ & $\mathrm{~S} * \mathrm{~T}$ & $\mathrm{~F}^{*} \mathrm{~T}$ & $\mathrm{~S}^{*} \mathrm{~F}^{*} \mathrm{~T}$ & \\
\hline & 0.13 & 0.12 & 0.10 & 0.22 & 0.26 & 0.20 & 0.45 & \\
\hline
\end{tabular}

a, b \& O: There is no significant difference $(\mathrm{P}>0.05)$ between any two means, within the same column have the same superscript letter. $n=40$. A, B \& O: There is no significant difference $(\mathrm{P}>0.05)$ between any two means for the same attribute within the same row have the same superscript letter. 
Table 10. Odor of orange-based formulated low calories jams sweetened with fructose, stevioside and sucralose during 12 months of storage (mean $\pm \mathrm{SD}$ ).

\begin{tabular}{|c|c|c|c|c|c|c|c|c|}
\hline \multirow{2}{*}{ Fruit type } & \multirow{2}{*}{$\begin{array}{l}\text { Sweetener } \\
\text { formula }\end{array}$} & \multicolumn{5}{|c|}{ Storage period (month) } & \multirow{2}{*}{$\begin{array}{l}\text { Mean of storage } \\
\text { period }\end{array}$} & \multirow{2}{*}{$\begin{array}{l}\text { Mean of } \\
\text { fruit type }\end{array}$} \\
\hline & & 0 & 3 & 6 & 9 & 12 & & \\
\hline \multirow{5}{*}{ Orange } & $\mathrm{O} 1$ & $5.33 \pm 0.92$ & $4.70 \pm 1.51$ & $4.3 \pm 1.02$ & $3.40 \pm 0.93$ & $3.43 \pm 0.93$ & $4.23 \pm 1.31 d$ & \multirow{5}{*}{$\begin{array}{l}3.91 \pm \\
1.26 \mathrm{~b}\end{array}$} \\
\hline & $\mathrm{O} 2$ & $5.00 \pm 0.99$ & $4.60 \pm 1.37$ & $4.1 \pm 1.06$ & $3.00 \pm 0.77$ & $3.00 \pm 0.77$ & $3.96 \pm 1.300$ & \\
\hline & $\mathrm{O} 3$ & $4.78 \pm 1.14$ & $4.20 \pm 1.18$ & $4.3 \pm 1.11$ & $3.10 \pm 0.71$ & $3.08 \pm 0.73$ & $3.89 \pm 1.200$ & \\
\hline & $\mathrm{O} 4$ & $4.44 \pm 0.75$ & $3.90 \pm 1.46$ & $4 \pm 0.64$ & $2.70 \pm 0.65$ & $2.70 \pm 0.65$ & $3.54 \pm 1.14 b$ & \\
\hline & O5 & $4.70 \pm 1.14$ & $4.70 \pm 1.36$ & $3.2 \pm 1.26$ & $3.40 \pm 0.93$ & $3.43 \pm 0.93$ & $3.89 \pm 1.310$ & \\
\hline \multirow{4}{*}{$\begin{array}{l}75 \% \text { orange/ } \\
25 \% \text { pumpkin }\end{array}$} & O6 & $4.95 \pm 1.02$ & $4.60 \pm 1.52$ & $3.7 \pm 1.02$ & $3.20 \pm 1.09$ & $3.20 \pm 1.09$ & $3.92 \pm 1.36 \mathrm{O}$ & \multirow{4}{*}{$\begin{array}{l}3.87 \pm \\
1.31 b\end{array}$} \\
\hline & $\mathrm{O} 7$ & $5.03 \pm 1.07$ & $4.30 \pm 1.64$ & $3.8 \pm 0.88$ & $3.30 \pm 0.46$ & $3.25 \pm 0.54$ & $3.94 \pm 1.200$ & \\
\hline & O8 & $4.73 \pm 1.45$ & $4.50 \pm 1.30$ & $3.8 \pm 1.26$ & $2.90 \pm 0.71$ & $2.85 \pm 0.70$ & $3.76 \pm 1.37 \mathrm{O}$ & \\
\hline & O9 & $3.95 \pm 1.14$ & $4.10 \pm 1.46$ & $3.1 \pm 0.96$ & $3.00 \pm 1.01$ & $3.00 \pm 0.96$ & $3.42 \pm 1.21 \mathrm{ab}$ & \\
\hline \multirow{3}{*}{$\begin{array}{l}75 \% \text { orange/ } \\
25 \% \text { papaya }\end{array}$} & $\mathrm{O} 10$ & $3.58 \pm 1.28$ & $4.40 \pm 1.22$ & $3.6 \pm 1.65$ & $2.90 \pm 1.15$ & $2.90 \pm 1.10$ & $3.48 \pm 1.40 \mathrm{ab}$ & \multirow{3}{*}{$\begin{array}{c}3.43 \pm \\
1.24 \mathrm{a}\end{array}$} \\
\hline & $\mathrm{O} 11$ & $4.03 \pm 1.07$ & $4.00 \pm 1.43$ & $3.5 \pm 1.52$ & $3.00 \pm 0.64$ & $3.00 \pm 0.64$ & $3.51 \pm 1.20 \mathrm{ab}$ & \\
\hline & $\mathrm{O} 12$ & $3.43 \pm 1.30$ & $4.40 \pm 1.13$ & $3.4 \pm 0.67$ & $2.70 \pm 0.79$ & $2.65 \pm 0.74$ & $3.32 \pm 1.14 \mathrm{a}$ & \\
\hline \multicolumn{2}{|c|}{ Mean of total storage period } & $4.49 \pm 1.250$ & $4.37 \pm 1.4 \mathrm{O}$ & $3.73 \pm 1.18 \mathrm{~B}$ & $3.05 \pm 0.86 \mathrm{~A}$ & $3.04 \pm 0.86 \mathrm{~A}$ & & \\
\hline \multicolumn{2}{|c|}{$\begin{array}{c}\text { Ratio of } \\
\text { (fructose:sweeteners) }\end{array}$} & $\begin{array}{c}\mathrm{O}(1,5,9) \\
(100 / 0)\end{array}$ & $\begin{array}{l}\mathrm{O}(2,6,10) \\
(75 / 25)\end{array}$ & $\begin{array}{l}\mathrm{O}(3,7,11) \\
(50 / 50)\end{array}$ & $\begin{array}{c}\mathrm{O}(4,8,12) \\
(25 / 75)\end{array}$ & & & \\
\hline \multicolumn{2}{|c|}{ Mean of sweetener formula } & $3.85 \pm 1.32 \mathrm{~B}$ & $3.78 \pm 1.37 \mathrm{~B}$ & $3.78 \pm 1.22 \mathrm{~B}$ & $3.54 \pm 1.23 \mathrm{~A}$ & & & \\
\hline \multirow{2}{*}{ LSD at 0.05 for: } & Storage (S) & Fruit (F) & Sweetener (T) & $\mathrm{S} * \mathrm{~F}$ & $\mathrm{~S} * \mathrm{~T}$ & $\mathrm{~F}^{*} \mathrm{~T}$ & $\mathrm{~S}^{*} \mathrm{~F}^{*} \mathrm{~T}$ & \\
\hline & 0.14 & 0.12 & 0.11 & 0.24 & 0.27 & 0.21 & 0.47 & \\
\hline
\end{tabular}

a, b \& O: There is no significant difference $(\mathrm{P}>0.05)$ between any two means, within the same column have the same superscript letter. $n=40$. A, B \& O: There is no significant difference $(\mathrm{P}>0.05)$ between any two means for the same attribute within the same row have the same superscript letter.

Table 11. Texture of orange-based formulated low calories jams sweetened with fructose, stevioside and sucralose during 12 months of storage (mean $\pm \mathrm{SD}$ ).

\begin{tabular}{|c|c|c|c|c|c|c|c|c|}
\hline \multirow{2}{*}{ Fruit type } & \multirow{2}{*}{$\begin{array}{l}\text { Sweetener } \\
\text { formula }\end{array}$} & \multicolumn{5}{|c|}{ Storage period (month) } & \multirow{2}{*}{$\begin{array}{c}\text { Mean of storage } \\
\text { period }\end{array}$} & \multirow{2}{*}{$\begin{array}{c}\text { Mean of fruit } \\
\text { type }\end{array}$} \\
\hline & & 0 & 3 & 6 & 9 & 12 & & \\
\hline \multirow{5}{*}{ Orange } & $\mathrm{O} 1$ & $4.88 \pm 1.51$ & $4 \pm 1.63$ & $3.9 \pm 1.39$ & $4.1 \pm 0.96$ & $4.08 \pm 0.97$ & $4.19 \pm 1.35$ Ode & \multirow{5}{*}{$4.16 \pm 1.33 b$} \\
\hline & $\mathrm{O} 2$ & $5.28 \pm 0.96$ & $4.4 \pm 1.93$ & $4 \pm 0.78$ & $3.68 \pm 1.32$ & $3.74 \pm 1.35$ & $4.23 \pm 1.44$ de & \\
\hline & $\mathrm{O} 3$ & $4.95 \pm 1.43$ & $4.3 \pm 1.81$ & $4.3 \pm 0.79$ & $3.9 \pm 0.96$ & $3.9 \pm 0.96$ & $4.27 \pm 1.29 \mathrm{de}$ & \\
\hline & $\mathrm{O} 4$ & $4.62 \pm 1.41$ & $4.7 \pm 1.36$ & $4.2 \pm 0.61$ & $3.1 \pm 0.55$ & $3.1 \pm 0.55$ & $3.94 \pm 1.20 \mathrm{~b}$ & \\
\hline & O5 & $5 \pm 1.34$ & $5.1 \pm 1.53$ & $3.6 \pm 0.67$ & $4 \pm 1.28$ & $4 \pm 1.24$ & $4.34 \pm 1.37 \mathrm{de}$ & \\
\hline \multirow{4}{*}{$\begin{array}{l}75 \% \text { orange/ } \\
25 \% \text { pumpkin }\end{array}$} & O6 & $5.51 \pm 1.21$ & $4.6 \pm 1.82$ & $3.8 \pm 0.88$ & $3.9 \pm 0.96$ & $3.8 \pm 1.04$ & $4.32 \pm 1.39 \mathrm{de}$ & \multirow{4}{*}{$4.41 \pm 1.34 \mathrm{c}$} \\
\hline & $\mathrm{O} 7$ & $5.75 \pm 1.01$ & $4.8 \pm 1.68$ & $4.2 \pm 0.76$ & $4.2 \pm 0.88$ & $4.08 \pm 1$ & $4.61 \pm 1.27 f$ & \\
\hline & $\mathrm{O} 8$ & $4.93 \pm 1.62$ & $4.9 \pm 1.84$ & $4.1 \pm 0.84$ & $4 \pm 0.78$ & $3.95 \pm 0.85$ & $4.38 \pm 1.33 \mathrm{ef}$ & \\
\hline & O9 & $5.03 \pm 1.28$ & $4.6 \pm 1.3$ & $4 \pm 0.78$ & $3.5 \pm 0.93$ & $3.45 \pm 1.01$ & $4.11 \pm 1.23 \mathrm{bOd}$ & \\
\hline \multirow{3}{*}{$\begin{array}{l}75 \% \text { orange/ } \\
25 \% \text { papaya }\end{array}$} & $\mathrm{O} 10$ & $4.83 \pm 0.98$ & $4.4 \pm 1.82$ & $4.2 \pm 0.76$ & $3.7 \pm 1.02$ & $3.65 \pm 1.08$ & $4.16 \pm 1.26$ bOde & \multirow{3}{*}{$3.98 \pm 1.40 \mathrm{a}$} \\
\hline & $\mathrm{O} 11$ & $4.95 \pm 1.18$ & $4.7 \pm 1.76$ & $3.5 \pm 1.22$ & $3.4 \pm 1.22$ & $3.35 \pm 1.23$ & $3.98 \pm 1.50 \mathrm{bO}$ & \\
\hline & $\mathrm{O} 12$ & $4.45 \pm 1.93$ & $3.7 \pm 1.87$ & $3 \pm 1.5$ & $3.6 \pm 0.93$ & $3.58 \pm 0.96$ & $3.67 \pm 1.56 a$ & \\
\hline \multirow{2}{*}{\multicolumn{2}{|c|}{$\begin{array}{c}\text { Mean of total storage period } \\
\text { Ratio of } \\
\text { (fructose:sweeteners) }\end{array}$}} & $5.01 \pm 1.38 \mathrm{D}$ & $4.52 \pm 1.730$ & $3.9 \pm 1.01 \mathrm{~B}$ & $3.76 \pm 1.04 \mathrm{AB}$ & $3.72 \pm 1.07 \mathrm{~A}$ & & \\
\hline & & $\begin{array}{l}\mathrm{O}(1,5,9) \\
(100 / 0)\end{array}$ & $\begin{array}{c}\mathrm{O}(2,6,10) \\
(75 / 25)\end{array}$ & $\begin{array}{c}\mathrm{O}(3,7,11) \\
(50 / 50)\end{array}$ & $\begin{array}{c}\mathrm{O}(4,8,12) \\
(25 / 75)\end{array}$ & & & \\
\hline \multicolumn{2}{|c|}{ Mean of sweetener formula } & $4.21 \pm 1.32 \mathrm{~B}$ & $4.23 \pm 1.36 \mathrm{~B}$ & $4.29 \pm 1.38 \mathrm{~B}$ & $3.99 \pm 1.40 \mathrm{~A}$ & & & \\
\hline \multirow{2}{*}{ LSD at 0.05 for: } & Storage (S) & Fruit (F) & Sweetener ( $\mathrm{T}$ ) & $\mathrm{S} * \mathrm{~F}$ & $\mathrm{~S} * \mathrm{~T}$ & $\mathrm{~F}^{*} \mathrm{~T}$ & $\mathrm{~S}^{*} \mathrm{~F}^{*} \mathrm{~T}$ & \\
\hline & 0.16 & 0.14 & 0.12 & 0.27 & 0.31 & 0.24 & 0.55 & \\
\hline
\end{tabular}

a, b \& O: There is no significant difference $(\mathrm{P}>0.05)$ between any two means, within the same column have the same superscript letter. $n=40$. A, B \& O: There is no significant difference $(\mathrm{P}>0.05)$ between any two means for the same attribute within the same row have the same superscript letter. 
Table 12. Bitterness of orange-based formulated low calories jams sweetened with fructose, stevioside and sucralose during 12 months of storage (mean $\pm \mathrm{SD}$ ).

\begin{tabular}{|c|c|c|c|c|c|c|c|c|}
\hline \multirow{2}{*}{ Fruit type } & \multirow{2}{*}{$\begin{array}{l}\text { Sweetener } \\
\text { formula }\end{array}$} & \multicolumn{5}{|c|}{ Storage period (month) } & \multirow{2}{*}{$\begin{array}{l}\text { Mean of storage } \\
\text { period }\end{array}$} & \multirow{2}{*}{$\begin{array}{l}\text { Mean of } \\
\text { fruit type }\end{array}$} \\
\hline & & 0 & 3 & 6 & 9 & 12 & & \\
\hline \multirow{5}{*}{ Orange } & $\mathrm{O} 1$ & $5.23 \pm 1.19$ & $5.2 \pm 0.88$ & $3.9 \pm 1.06$ & $3.1 \pm 0.84$ & $3.08 \pm 0.83$ & $4.10 \pm 1.36 \mathrm{bO}$ & \multirow{4}{*}{$\begin{array}{c}4.02 \pm \\
1.34^{\mathrm{a}}\end{array}$} \\
\hline & $\mathrm{O} 2$ & $5.13 \pm 1.3$ & $5 \pm 1.2$ & $4.3 \pm 0.65$ & $2.76 \pm 1.1$ & $2.92 \pm 1.24$ & $4.05 \pm 1.50 \mathrm{bO}$ & \\
\hline & $\mathrm{O} 3$ & $5.03 \pm 1.1$ & $4.9 \pm 1.06$ & $4.1 \pm 0.71$ & $3.2 \pm 0.88$ & $3.2 \pm 0.94$ & $4.09 \pm 1.23 \mathrm{bO}$ & \\
\hline & $\mathrm{O} 4$ & $4.49 \pm 1.32$ & $5.1 \pm 1.24$ & $3.2 \pm 1.09$ & $3.2 \pm 0.41$ & $3.23 \pm 0.53$ & $3.84 \pm 1.27 \mathrm{a}$ & \\
\hline & O5 & $5.35 \pm 1.25$ & $4.7 \pm 1.29$ & $3.7 \pm 1.2$ & $3.6 \pm 1.03$ & $3.6 \pm 1.01$ & $4.19 \pm 1.35 b O d$ & \multirow{5}{*}{$\begin{array}{l}4.29 \pm \\
1.36 \mathrm{c}\end{array}$} \\
\hline \multirow{4}{*}{$\begin{array}{l}75 \% \text { orange/ } \\
25 \% \text { pumpkin }\end{array}$} & O6 & $5.62 \pm 0.91$ & $5.3 \pm 0.79$ & $4.5 \pm 0.82$ & $3.1 \pm 0.84$ & $3.2 \pm 0.94$ & $4.34 \pm 1.35$ de & \\
\hline & $\mathrm{O} 7$ & $5.63 \pm 0.87$ & $5 \pm 1.11$ & $4.5 \pm 0.82$ & $3.6 \pm 1.13$ & $3.6 \pm 1.15$ & $4.47 \pm 1.29 \mathrm{e}$ & \\
\hline & O8 & $5.58 \pm 0.84$ & $5.2 \pm 1.49$ & $3.7 \pm 0.91$ & $3.2 \pm 0.88$ & $3.25 \pm 0.93$ & $4.19 \pm 1.44 \mathrm{bOd}$ & \\
\hline & O9 & $5.05 \pm 1.21$ & $4.9 \pm 1.24$ & $3.9 \pm 1.15$ & $3.3 \pm 0.91$ & $3.33 \pm 0.89$ & $4.09 \pm 1.31 \mathrm{bO}$ & \\
\hline \multirow{3}{*}{$\begin{array}{l}75 \% \text { orange/ } \\
25 \% \text { papaya }\end{array}$} & $\mathrm{O} 10$ & $5.63 \pm 0.63$ & $4.8 \pm 0.99$ & $4.7 \pm 1.02$ & $3 \pm 0.91$ & $3.05 \pm 0.9$ & $4.24 \pm 1.37 \mathrm{Od}$ & \multirow{3}{*}{$\begin{array}{l}4.14 \pm \\
1.34 \mathrm{~b}\end{array}$} \\
\hline & $\mathrm{O} 11$ & $5.6 \pm 0.78$ & $5 \pm 1.2$ & $3.9 \pm 1.06$ & $3.3 \pm 0.91$ & $3.3 \pm 0.94$ & $4.22 \pm 1.35 b O d$ & \\
\hline & $\mathrm{O} 12$ & $5.15 \pm 1.1$ & $5 \pm 1.2$ & $3.7 \pm 0.79$ & $3.1 \pm 0.96$ & $3.15 \pm 0.98$ & $4.02 \pm 1.34 \mathrm{ab}$ & \\
\hline \multicolumn{2}{|c|}{ Mean of total storage period } & $5.29 \pm 1.10 \mathrm{D}$ & $5.01 \pm 1.15 \mathrm{O}$ & $4.01 \pm 1.03 \mathrm{~B}$ & $3.21 \pm 0.93 \mathrm{~A}$ & $3.24 \pm 0.96 \mathrm{~A}$ & & \\
\hline \multicolumn{2}{|c|}{$\begin{array}{c}\text { Ratio of } \\
\text { (fructose:sweeteners) }\end{array}$} & $\begin{array}{l}\mathrm{O}(1,5,9) \\
(100 / 0)\end{array}$ & $\begin{array}{l}\mathrm{O}(2,6,10) \\
(75 / 25)\end{array}$ & $\begin{array}{l}\mathrm{O}(3,7,11) \\
(50 / 50)\end{array}$ & $\begin{array}{l}\mathrm{O}(4,8,12) \\
(25 / 75)\end{array}$ & & & \\
\hline \multicolumn{2}{|c|}{ Mean of sweetener formula } & $4.13 \pm 1.34 \mathrm{AB}$ & $4.21 \pm 1.41 \mathrm{BC}$ & $4.26 \pm 1.3 \mathrm{C}$ & $4.02 \pm 1.36 \mathrm{~A}$ & & & \\
\hline \multirow{2}{*}{ LSD at 0.05 for: } & Storage (S) & Fruit (F) & Sweetener ( $\mathrm{T}$ ) & $\mathrm{S} * \mathrm{~F}$ & $\mathrm{~S}^{*} \mathrm{~T}$ & $\mathrm{~F}^{*} \mathrm{~T}$ & $\mathrm{~S}^{*} \mathrm{~F}^{*} \mathrm{~T}$ & \\
\hline & 0.13 & 0.11 & 0.10 & 0.22 & 0.26 & 0.20 & 0.44 & \\
\hline
\end{tabular}

a, b \& O: There is no significant difference $(\mathrm{P}>0.05)$ between any two means, within the same column have the same superscript letter. $n=40$. A, B \& O: There is no significant difference $(\mathrm{P}>0.05)$ between any two means for the same attribute within the same row have the same superscript letter.

[35] showed that formulations containing 3.7\% to $37.7 \%$ papaya in mixed fruit jam produced optimum acceptance for color. A decremental rate in color was monitored during 12 months. This result was in accordance with Ehsan et al. [70] who reported decrease in color during storage of grape fruit apple marmalade. Replacing fructose by St \& Su improved the color significantly. This result was in agreement with Youssef et al. [32]. Also, Carvalho et al. [71] showed that used of Reb-A as a sweetener in strawberry diet jam was given a bitter sensory performance.

The obtained data in Table 9 indicated that the highest score was recorded for OK jams (3.86 \pm 1.35$)$ followed by orange jams $(3.76 \pm 1.29)$ while OY jams recorded the lowest score (3.54 \pm 1.26$)$, significantly. During the storage, the jams taste was deteriorated significantly. This result was in agreement with Bajwa et al. [72]. Concerning the mean of sweetener formulas, results indicated that maximum mean score was $3.82 \pm 1.28$ obtained by ratio of (50:50). These results were in agreement with Kerdsup et al. [73] and Gajar et al. [74].

Oder of formulated low caloric jams was tabulated in Table 10. The obtained data indicated that no significant $(\mathrm{P}<0.05)$ difference was found between OK and orange jam while a significant differences between them and OY and both OK and orange jams was recorded. Replacing the fructose with St \& Su did not affect the odor even at $50 \%$. As shown in other sensory attributes the odor also deteriorated with prolong of storage period. The replacing of $75 \%$ of fructose affect the odor may be due to the effect of cooking temperature on flavor substances which affecting the odor. These results are in harmony with Gajar et al. [74] and Carvalho et al. [71].

In Table 11, there is a significant difference $(\mathrm{P}<0.05)$ was found among all formulated orange jams. After one year storage, results concluded that OK jam formulas was the better than orange or OY jams. These results are in accordance with confirmed results by [52]. A decremental rate in jam texture was correlated to increasing of storage period. This result is agreement with Ehsan et al. [70] whom recorded a decrease in texture from 8.80 to 7.96 in grape fruit apple marmalade during storage. Also, Muhammad et al. [75] reported that the mean score for texture significantly decreased from 9.00 to 6.7 during storage in apple diet jam. Regarding the general mean 
of sweetener formulas the results indicated no significant differences between the ratios (100:0), (75:25), and (50:50) while there is a significant difference between ratio (25:75) and all replacing ratios confirming the texture degradation in low fructose jams [52] [75].

The Bitterness characteristic of sweetened jam with fructose, St and Su was illustrated in Table 12. Comparing the fruits used in jam making a significant difference $(\mathrm{P}<0.05)$ was observed among the group formulas recorded the lowest bitterness as high scored for OY jams while the lowest score was given to orange as highest bitterness was recorded. During the storage period, an incremental rate in bitterness was observed when monitoring the mean of total storage period. Comparing between mean of sweetener formulas the results indicated that maximum mean score for bitterness was $4.26 \pm 1.3$ obtained by ratio (50:50). A non-significant difference was observed between different sweetened jams; means that addition of different Su and St for producing diet jams don not affect the taste. These results are in agreement with Khouryieh et al. [76] and Prakash et al. [77].

\section{Conclusion}

The use of sweeteners such as fructose, sucralose and stevioside in the manufacture of orange diet jam was shown to be satisfactory, resulting in a product with jam characteristics and with flavor, taste and texture similar to conventional jam, with low caloric value, allowing its indication as much for diabetics as to the individuals that are on a diet with caloric restriction. This type of product can be recommended as an antioxidant booster for the consumers in health point of view. The product can be safely consumed up to a period of 12 months without any deterioration in its quality at room temperature condition.

\section{References}

[1] Weaver, D. and Finke, M. (2003) The Relationship between the Use of Sugar Content Information on Nutrition Labels and the Consumption of Added Sugars. Food Policy, 28, 213-219. http://dx.doi.org/10.1016/S0306-9192(03)00028-9

[2] Dietz, W.H., Benken, D.E. and Hunter, A.S. (2009) Public Health Law and the Prevention and Control of Obesity. Milbank Quarterly, 87, 215-227. http://dx.doi.org/10.1111/j.1468-0009.2009.00553.x

[3] Silver, L. and Bassett, M.T. (2008) Food Safety for the 21st Century. JAMA, 300, 957-959. http://dx.doi.org/10.1001/jama.300.8.957

[4] Brownell, K.D., Schwartz, M.B., Puhl, R.M., Henderson, K.E. and Harris, J.L. (2009) The Need for Bold Action to Prevent Adolescent Obesity. Journal of Adolescent Health, 45, S8-S17. http://dx.doi.org/10.1016/j.jadohealth.2009.03.004

[5] Dabelea, D., Bell, R.A., D’Agostino Jr, R.B., Imperatore, G., Johansen, J.M., Linder, B., Liu, L.L., Loots, B., Marcovina, S. and Mayer-Davis, E.J. (2007) Incidence of Diabetes in Youth in the United States. JAMA: The Journal of the American Medical Association, 297, 2716-2724. http://dx.doi.org/10.1001/jama.297.24.2716

[6] Ogden, C.L., Carroll, M.D., Curtin, L.R., McDowell, M.A., Tabak, C.J. and Flegal, K.M. (2006) Prevalence of Overweight and Obesity in the United States, 1999-2004. JAMA, 295, 1549-1555. http://dx.doi.org/10.1001/jama.295.13.1549

[7] Cardoso, J.M.P. and Bolini, H.M.A. (2007) Different Sweeteners in Peach Nectar: Ideal and Equivalent Sweetness. Food Research International, 40, 1249-1253. http://dx.doi.org/10.1016/j.foodres.2007.08.004

[8] Pinheiro, M., Oliveira, M., Penna, A. and Tamime, A. (2005) The Effect of Different Sweeteners in Low-Calorie Yogurts-A Review. International Journal of Dairy Technology, 58, 193-199. http://dx.doi.org/10.1111/j.1471-0307.2005.00228.x

[9] Fernandes, F.A., Gallão, M.I. and Rodrigues, S. (2008) Effect of Osmotic Dehydration and Ultrasound Pre-Treatment on Cell Structure: Melon Dehydration. LWT-Food Science and Technology, 41, 604-610. http://dx.doi.org/10.1016/j.lwt.2007.05.007

[10] Wiebe, N., Padwal, R., Field, C., Marks, S., Jacobs, R. and Tonelli, M. (2011) A Systematic Review on the Effect of Sweeteners on Glycemic Response and Clinically Relevant Outcomes. BMC Medicine, 9, 123. http://dx.doi.org/10.1186/1741-7015-9-123

[11] Mattes, R.D. and Popkin, B.M. (2009) Nonnutritive Sweetener Consumption in Humans: Effects on Appetite and Food Intake and Their Putative Mechanisms. The American Journal of Clinical Nutrition, 89, 1-14. http://dx.doi.org/10.3945/ajcn.2008.26792

[12] Fitch, C. and Keim, K.S. (2012) Position of the Academy of Nutrition and Dietetics: Use of Nutritive and Nonnutritive Sweeteners. Journal of the Academy of Nutrition and Dietetics, 112, 739-758. http://dx.doi.org/10.1016/j.jand.2012.03.009 
[13] UK, D. (2012) Number of People Diagnosed with Diabetes Reaches Three Million.

[14] Bellisle, F. and Drewnowski, A. (2007) Intense Sweeteners, Energy Intake and the Control of Body Weight. European Journal of Clinical Nutrition, 61, 691-700. http://dx.doi.org/10.1038/sj.ejcn.1602649

[15] BSDA (2013) Vitamins and Minerals.

[16] Binns, N.M. (2003) Sucralose-All Sweetness and Light. Nutrition Bulletin, 28, 53-58. http://dx.doi.org/10.1046/j.1467-3010.2003.00307.x

[17] de Arruda, J.G.F., Martins, A.T. and Azoubel, R. (2003) Ciclamato de sódio e rim fetal. Revista Brasileira de Saúde Materno Infantil, 3, 147-150. http://dx.doi.org/10.1590/s1519-38292003000200003

[18] Portela, G. and Azoubel, R. (2004) Nefrotoxicidade fetal com o uso da amicacina. Estudo cariométrico. Jornal Brasileiro de Nefrologia, 26, 12-18.

[19] Martins, A.T., Azoubel, R., Lopes, R.A., di Matteo, M.A.S. and de Arruda, J.G.F. (2005) Efectos del ciclamato de sódio en el hígado fetal de ratas: estudios cariométrico y estereológico. International Journal of Morphology, 23, 221226.

[20] de Matos, M.A., Martins, A.T. and Azoubel, R. (2006) Efectos del ciclamato de sodio en la placenta de rata: Estudio morfométrico. International Journal of Morphology, 24, 137-142. http://dx.doi.org/10.4067/S0717-95022006000300001

[21] Fetal, H. (2007) Effects of Aspartame on Maternal-Fetal and Placental Weights, Length of Umbilical Cord and Fetal Liver: A Kariometric Experimental Study. International Journal of Morphology, 25, 549-554.

[22] Lemus-Mondaca, R., Vega-Gálvez, A., Zura-Bravo, L. and Ah-Hen, K. (2012) Stevia rebaudiana Bertoni, Source of a High-Potency Natural Sweetener: A Comprehensive Review on the Biochemical, Nutritional and Functional Aspects. Food Chemistry, 132, 1121-1132. http://dx.doi.org/10.1016/j.foodchem.2011.11.140

[23] Shah, A.B., Jones, G.P. and Vasiljevic, T. (2010) Sucrose-Free Chocolate Sweetened with Stevia rebaudiana Extract and Containing Different Bulking Agents-Effects on Physicochemical and Sensory Properties. International Journal of Food Science \& Technology, 45, 1426-1435. http://dx.doi.org/10.1111/j.1365-2621.2010.02283.x

[24] JFECFA (2004) Sixty-Third Meeting. WHO, Geneva.

[25] Ghanta, S., Banerjee, A., Poddar, A. and Chattopadhyay, S. (2007) Oxidative DNA Damage Preventive Activity and Antioxidant Potential of Stevia rebaudiana (Bertoni) Bertoni, a Natural Sweetener. Journal of Agricultural and Food Chemistry, 55, 10962-10967. http://dx.doi.org/10.1021/jf071892q

[26] Muhammad, A., Durrani, Y., Zeb, A., Ayub, M. and Ullah, J. (2008) Development of Diet Jam from Apple Grown in Swat (NWFP). Sarhad Journal of Agriculture, 24, 461-467.

[27] Kopjar, M., Pilizota, V., Tiban, N.N., Subaric, D., Babic, J., Ackar, D. and Sajdl, M. (2009) Strawberry Jams: Influence of Different Pectins on Colour and Textural Properties. Czech Journal of Food Sciences, 27, 20-28.

[28] Tamer, C.E., Incedayi, B., Yönel, S.P., Yonak, S. and Copur, O.U. (2010) Evaluation of Several Quality Criteria of Low Calorie Pumpkin Dessert. Notulae Botanicae Horti Agrobotanici Cluj-Napoca, 38, 76-80.

[29] Basu, S., Shivhare, U., Singh, T. and Beniwal, V. (2011) Rheological, Textural and Spectral Characteristics of Sorbitol Substituted Mango Jam. Journal of Food Engineering, 105, 503-512. http://dx.doi.org/10.1016/j.jfoodeng.2011.03.014

[30] Gao, X., Yu, T., Zhang, Z.-H., Xu, J.-C. and Fu, X.-T. (2011) Rheological and Sensory Properties of Four Kinds of Jams. Journal of Stored Products and Postharvest Research, 2, 227-234.

[31] Kerdsup, P. and Naknean, P. (2013) Effect of Sorbitol Substitution on Physical, Chemical and Sensory Properties of Low Sugar Mango Jam. International Scientific Proceedings, 13, 12-18.

[32] Youssef, H.M. and Mousa, R.M. (2012) Nutritional Assessment of Low-Calorie Baladi Rose Petals Jam. Food and Public Health, 2, 197-201.

[33] Teangpook, C. and Paosantong, U. (2013) Production and Shelf-Life of Low Sucrose Lime Juice Papaya Jam. Pakistan Journal of Nutrition, 12, 870-878. http://dx.doi.org/10.3923/pjn.2013.870.878

[34] Levaj, B., Dragović-Uzelac, V., Dančević, T., Liber, S., Repajić, M. and Bursać Kovačević, D. (2009) Quality of Clementine Jam Influenced by Purée Pretreatment, Sugar Type and Pectin Addition. Agriculturae Conspectus Scientificus (ACS), 74, 227-231.

[35] Abdullah, A. and Cheng, T.C. (2001) Optimization of Reduced Calorie Tropical Mixed Fruits Jam. Food Quality and Preference, 12, 63-68. http://dx.doi.org/10.1016/S0950-3293(00)00030-6

[36] AOAC (2005) Official Methods of Analysis of AOAC International. 18th Edition, AOAC International, Gaithersburg.

[37] Wettestein, D.V. (1957) Chlorophyll-letale und der submikroskopische Formwechsel der Plastiden. Experimental Cell Research, 12, 427-506. http://dx.doi.org/10.1016/0014-4827(57)90165-9

[38] Ough, C.S. and Amerine, M.A. (1988) Methods for Analysis of Musts and Wines. Wiley-Interscience, New York, 377 p. 
[39] Zhang, D. and Hamauzu, Y. (2004) Phenolics, Ascorbic Acid, Carotenoids and Antioxidant Activity of Broccoli and Their Changes during Conventional and Microwave Cooking. Food Chemistry, 88, 503-509. http://dx.doi.org/10.1016/j.foodchem.2004.01.065

[40] Wilson, C.D., Pace, E., Bromfield, G., Jones, J.Y. and Lu, J.Y. (1998) Consumer Acceptance of Vegetarian Sweet Potato Products Intended for Space Missions. Life Support \& Biosphere Science, 5, 339-345.

[41] Steel, R., Torrie, J. and Dickey, D. (1997) Principles and Procedures of Statistics: A Biometrical Approach. 3rd Edition, McGraw-Hill, New York.

[42] Salvador, A.C., Dantas, M.I. de S., Vasconcelos, C.M., Vanetti, M.C.D., Ribeiro, S.M.R., Nery-Enes, B., Nelson, J.D. and Martino, H.S.D. (2012) Development of a Low Calorie Yacon Jam: Physico Chemical, Microbiological and Sensory Characterization. Revista chilena de nutrición, 39, 72-77.

[43] Mahan, L.K. and Alimentos, E.S.S. (2005) Nutrição e dietoterapia. Roca, São Paulo, 341-356.

[44] Yuyama, L.K.O., Pantoja, L., Maeda, R.N., Aguiar, J.P.L. and da Silva, S.B. (2008) Development and Acceptability of a Low Calorie Cubiu (Solanum sessiliflorum Dunal) Jam. Food Science and Technology (Campinas), 28, 929-934. http://dx.doi.org/10.1590/S0101-20612008000400026

[45] Chatsudthipong, V. and Muanprasat, C. (2009) Stevioside and Related Compounds: Therapeutic Benefits beyond Sweetness. Pharmacology \& Therapeutics, 121, 41-54. http://dx.doi.org/10.1016/j.pharmthera.2008.09.007

[46] Thompson, J., Drake, M., Lopetcharat, K. and Yates, M. (2004) Preference Mapping of Commercial Chocolate Milks. Journal of Food Science, 69, S406-S413. http://dx.doi.org/10.1111/j.1365-2621.2004.tb09958.x

[47] Le, K., Chiu, F. and Ng, K. (2007) Identification and Quantification of Antioxidants in Fructus lycii. Food Chemistry, 105, 353-363. http://dx.doi.org/10.1016/j.foodchem.2006.11.063

[48] Istratii, D., Vizireanu, C., Iordachescu, G., Dima, F. and Garnai, M. (2013) Physico-Chemical Characteristics and Antioxidant Activity of Goji Fruits Jam and Jelly during Storage. Annals of the University Dunarea de Jos of Galati Fascicle VI-Food Technology, 37, 100-110.

[49] Corrêa, R.C., Haminiuk, C.W., Sora, G.T., Bergamasco, R. and Vieira, A. (2014) Antioxidant and Rheological Properties of Guava Jam with Added Concentrated Grape Juice. Journal of the Science of Food and Agriculture, 94, 146-152. http://dx.doi.org/10.1002/jsfa.6233

[50] Poiana, M.-A., Alexa, E. and Mateescu, C. (2012) Tracking Antioxidant Properties and Color Changes in Low-Sugar Bilberry Jam as Effect of Processing, Storage and Pectin Concentration. Chemistry Central Journal, 6.

[51] Rababah, T.M., Al-U’Datt, M., Al-Mahasneh, M., Yang, W., Feng, H., Ereifej, K., Kilani, I. and Ishmais, M.A. (2014) Effect of Jam Processing and Storage on Phytochemicals and Physiochemical Properties of Cherry at Different Temperatures. Journal of Food Processing and Preservation, 38, 247-254. http://dx.doi.org/10.1002/jsfa.6233

[52] Kopjar, M., Pilizota, V., Tiban, N.N., Subaric, D., Babic, J., Ackar, D. and Sajdl, M. (2009) Strawberry Jams: Influence of Different Pectins on Color and Textural Properties. Czech Journal of Food Sciences, 27, 20-28.

[53] Shukla, S., Mehta, A., Bajpai, V.K. and Shukla, S. (2009) In Vitro Antioxidant Activity and Total Phenolic Content of Ethanolic Leaf Extract of Stevia rebaudiana Bert. Food and Chemical Toxicology, 47, 2338-2343. http://dx.doi.org/10.1016/j.fct.2009.06.024

[54] Tadhani, M., Patel, V. and Subhash, R. (2007) In Vitro Antioxidant Activities of Stevia rebaudiana Leaves and Callus. Journal of Food Composition and Analysis, 20, 323-329. http://dx.doi.org/10.1016/j.jfca.2006.08.004

[55] Schmidt, B.M., Erdman, J.W. and Lila, M.A. (2005) Effects of Food Processing on Blueberry Antiproliferation and Antioxidant Activity. Journal of Food Science, 70, s389-s394. http://dx.doi.org/10.1111/j.1365-2621.2005.tb11461.x

[56] Šavikin, K., Zdunić, G., Janković, T., Tasić, S., Menković, N., Stević, T. and Đorđević, B. (2009) Phenolic Content and Radical Scavenging Capacity of Berries and Related Jams from Certificated Area in Serbia. Plant Foods for Human Nutrition, 64, 212-217. http://dx.doi.org/10.1007/s11130-009-0123-2

[57] Scibisz, I. and Mitek, M. (2009) Effect of Processing and Storage Conditions on Phenolic Compounds and Antioxidant Capacity of Highbush Blueberry Jams. Polish Journal of Food and Nutrition Sciences, 59, 45-52.

[58] Howard, L.R., Castrodale, C., Brownmiller, C. and Mauromoustakos, A. (2010) Jam Processing and Storage Effects on Blueberry Polyphenolics and Antioxidant Capacity. Journal of Agricultural and Food Chemistry, 58, 4022-4029. http://dx.doi.org/10.1021/jf902850h

[59] Kamiloglu, S., Pasli, A.A., Ozcelik, B., Van Camp, J. and Capanoglu, E. (2015) Color Retention, Anthocyanin Stability and Antioxidant Capacity in Black Carrot (Daucus carota) Jams and Marmalades: Effect of Processing, Storage Conditions and in Vitro Gastrointestinal Digestion. Journal of Functional Foods, 13, 1-10. http://dx.doi.org/10.1016/j.jff.2014.12.021

[60] Kandlakunta, B., Rajendran, A. and Thingnganing, L. (2008) Carotene Content of Some Common (Cereals, Pulses, Vegetables, Spices and Condiments) and Unconventional Sources of Plant Origin. Food Chemistry, 106, 85-89. 
http://dx.doi.org/10.1016/j.foodchem.2007.05.071

[61] Li, Y. and Schellhorn, H.E. (2007) New Developments and Novel Therapeutic Perspectives for Vitamin C. The Journal of Nutrition, 137, 2171-2184.

[62] Johnson, J., Abam, K., Ujong, U., Odey, M., Inekwe, V., Dasofunjo, K. and Inah, G. (2013) Vitamins Composition of Pulp, Seed and Rind of Fresh and Dry Rambutan Nephelium Lappaceum and Squash Cucurbita pepo L. International Journal of Science and Technology, 2, 71-76.

[63] Nwofia, G.E., Ojimelukwe, P. and Eji, C. (2012) Chemical Composition of Leaves, Fruit Pulp and Seeds in Some Carica papaya (L) Morphotypes. International Journal of Medicinal and Aromatic Plants, 2, 200-206.

[64] Selvamuthukumaran, M. and Khanum, F. (2014) Processing Seabuckthorn Fruit for Antioxidant Rich Jam Development and Shelf Stability Assessment. Indian Journal of Traditional Knowledge, 13, 335-346.

[65] Touati, N., Tarazona-Díaz, M.P., Aguayo, E. and Louaileche, H. (2014) Effect of Storage Time and Temperature on the Physicochemical and Sensory Characteristics of Commercial Apricot Jam. Food Chemistry, 145, 23-27. http://dx.doi.org/10.1016/j.foodchem.2013.08.037

[66] Safdar, M.N., Mumtaz, A., Hameed, T., Siddiqui, N., Khalil, S. and Amjad, M. (2012) Storage Studies of Jam Prepared from Different Mango Varieties. Pakistan Journal of Nutrition, 11, 555-561. http://dx.doi.org/10.3923/pjn.2012.653.659

[67] Sindumathi, G. and Amutha, S. (2014) Processing and Quality Evaluation of Coconut Based Jam. IOSR Journal of Environmental Science, Toxicology and Food Technology, 8, 10-14. http://dx.doi.org/10.9790/2402-08111014

[68] Mehta, U. and Bajaj, S. (1983) Effects of Storage and Methods of Preservation on the Physico-Chemical Characteristics of Citrus Juices. Indian Food Packer, 37, 42-51.

[69] Joy, K. (2013) Formulation, Sensory Evaluation and Nutrient Analysis of Products with Aloe Vera. World Journal of Pharmacy and Pharmaceutical Sciences, 2, 5321-5328.

[70] Ehsan, E., Naeem, Z., Javed, A. and Nazir, A. (2003) Development, Standardization and Storage Studies on Grape Fruit Apple Marmalade. Pakistan Journal of Food Sciences (Pakistan), 13, 11-15.

[71] de Carvalho, A.C.G., de Oliveira, R.C.G., Navacchi, M.F.P., da Costa, C.E.M., Mantovani, D., Dacôme, A.S., Seixas, F.A.V. and da Costa, S.C. (2013) Evaluation of the Potential Use of Rebaudioside-A as Sweetener for Diet Jam. Food Science and Technology (Campinas), 33, 555-560. http://dx.doi.org/10.1590/S0101-20612013005000080

[72] Bajwa, E.E., Parwaz, N.Z. and Ghafoor, A. (2002) Development, Standardization and Storage Studies on WatermelonLemon Jam. Pakistan Journal of Food Sciences (Pakistan), 12, 21-24.

[73] Kerdsup, P. and Naknean, P. (2013) Effect of Sorbitol Substitution on Physical, Chemical and Sensory Properties of Low Sugar Mango Jam. International Scientific Proceeding, 13, 12-18.

[74] Gajar, A.M. and Badrie, N. (2002) Processing and Quality Evaluation of a Low-Calorie Christophene Jam (Sechium edule (Jacq.) Swartz. Journal of Food Science, 67, 341-346. http://dx.doi.org/10.1111/j.1365-2621.2002.tb11407.x

[75] Muhammad, A., Durrani, Y., Ayub, M., Zeb, A. and Ullah, J. (2009) Organoleptic Evaluation of Diet Apple Jam from Apple Grown in Swat Valley. Sarhad Journal of Agriculture, 25, 81-86.

[76] Khouryieh, H.A., Aramouni, F.M. and Herald, T.J. (2005) Physical, Chemical and Sensory Properties of Sugar-Free Jelly. Journal of Food Quality, 28, 179-190. http://dx.doi.org/10.1111/j.1745-4557.2005.00014.x

[77] Prakash, I., DuBois, G., Clos, J., Wilkens, K. and Fosdick, L. (2008) Development of Rebiana, a Natural, Non-Caloric Sweetener. Food and Chemical Toxicology, 46, S75-S82. http://dx.doi.org/10.1016/j.fct.2008.05.004 